\title{
Endogenous Specialized Proresolving Mediator Profiles in a Novel Experimental Model of Lymphatic Obstruction and Intestinal Inflammation in African Green Monkeys
}

Felix Becker, ${ }^{* \dagger}$ Emily Romero, ${ }^{\ddagger}$ Jason Goetzmann, ${ }^{\ddagger}$ Dana L. Hasselschwert, ${ }^{\ddagger}$ Beth Dray, ${ }^{\S}$ John Vanchiere, ${ }^{\natural}$ Jane Fontenot, J. Winny Yun, ${ }^{*}$ Paul C. Norris, ${ }^{\prime}$ Luke White, ${ }^{*}$ Melany Musso, ${ }^{\ddagger}$ Charles N. Serhan, $"$ J. Steven Alexander, ${ }^{* * *}$ and Felicity N.E. Gavins* ${ }^{* \dagger}$

Accepted for publication May 9, 2019.

Address correspondence to Felicity N.E. Gavins, Ph.D., Department of Molecular and Cellular Physiology, Louisiana State University Health Sciences Center-Shreveport, 1501 Kings Hwy., Shreveport, LA 71130. E-mail: fgavin@ lsuhsc.edu.

\begin{abstract}
Changes in the intestinal lymphatic vascular system, such as lymphatic obstruction, are characteristic features of inflammatory bowel diseases. The lymphatic vasculature forms a conduit to enable resolution of inflammation; this process is driven by specialized endogenous proresolving mediators (SPMs). To evaluate contributions of lymphatic obstruction to intestinal inflammation and to study profiles of SPMs, we generated a novel animal model of lymphatic obstruction using African green monkeys. Follow-up studies were performed at 7, 21, and 61 days. Inflammation was determined by histology. Luminex assays were performed to evaluate chemokine and cytokine levels. In addition, lipid mediator metabololipidomic profiling was performed to identify SPMs. After 7 days, lymphatic obstruction resulted in a localized inflammatory state, paralleled by an increase in inflammatory chemokines and cytokines, which were found to be up-regulated after 7 days but returned to baseline after 21 and 61 days. At the same time, a distinct pattern of SPMs was profiled, with an increase for D-series resolvins, protectins, maresins, and lipoxins at 61 days. These results indicate that intestinal lymphatic obstruction can lead to an acute inflammatory state, accompanied by an increase in proinflammatory mediators, followed by a phase of resolution, paralleled by an increase and decrease of respective SPMs. (Am J Pathol 2019, 189: 1953-1972; https://doi.org/10.1016/j.ajpath.2019.05.013)
\end{abstract}

Inflammatory bowel diseases (IBDs), including its two main entities [Crohn disease (CD) and ulcerative colitis (UC)], represent chronic relapsing immune-mediated inflammatory disorders of the gastrointestinal tract. Despite a worldwide increase in prevalence and incidence, the exact etiology is still unknown. The current concept of IBD etiopathogenesis is that in genetically susceptible individuals, environmental factors and a dysregulated intestinal microbiome initiate an inappropriate inflammatory response by the host's mucosal immunity, leading to chronic intestinal inflammation. ${ }^{1}$ This multifactorial hypothesis is based on the complex interplay of various immune and nonimmune cells within the mucosal immune system.

Supported by the NIH/National Heart, Lung, and Blood Institute grant 1R01HL134959-01A1 (F.G.); Feist Weiler Cancer Center, Louisiana State University Shreveport, institutional funding (J.S.A. and F.B.); and the US Department of Defense grant PR100451 (J.S.A). The experiments in Boston, MA, were supported by NIH grant P01GM095467 (C.N.S.).

J.S.A. and F.N.E.G. contributed equally to this work.

Disclosures: None declared. 
Among these nonimmune components, intestinal lymphatic vessels have gained recent attention among the IBD research community, especially for $\mathrm{CD} .{ }^{2}$ The intestinal lymphatic vasculature is a unidirectional drainage system for interstitial fluids, lipids, small soluble antigens, and perivascularly infiltrated immune cells, organized into a network of capillaries and collecting vessels. ${ }^{3,4}$ The intestine contains lymphatic capillaries, whereas in the mesentery, only collecting vessels can be found, which run parallel with mesenteric arteries and veins. ${ }^{3}$ Studies in humans have revealed structural and functional alterations in the intestinal lymphatic vasculature in $\mathrm{CD} .^{5}$ Indeed, among the long recognized fundamental histopathologic hallmarks of $\mathrm{CD}$ are intralymphatic lymphocyte stasis, lymphangiectasia, and inflammatory granulomas resembling a chronic lymphocytic and granulomatous lymphangitis. ${ }^{2}$ Using modern imaging techniques, a growing body of evidence points toward lymphatic expansion and altered lymphatic drainage in the small and large intestine of CD patients. ${ }^{6-9}$ Recently, Randolph et al $^{10}$ presented similar findings of lymphatic dysfunction for larger mesenteric collecting vessels, suggesting a CD-associated reduction in the access of antigens and immune cells to mesenteric lymph nodes, potentially linking dysfunction of the intestinal lymphatic system and impaired immunity in CD. Concomitantly, murine models have provided further evidence and unraveled molecular mechanisms for a key role of lymphatic defects in the initiation and perpetuation of intestinal inflammation. Blocking intestinal inflammation-associated lymphangiogenesis has been shown to result in reduced vessel density and decreased intestinal lymph flow, which subsequently causes an attenuated antigen clearance, all of which exacerbated experimental colitis. ${ }^{11-15}$ In agreement with this, promotion of intestinal inflammation-associated lymphangiogenesis via adenoviral induction of vascular endothelial growth factor-C improved lymph flow, immune cell drainage, and bacterial antigen clearance, which resulted in diminished intestinal inflammation. ${ }^{12}$ This growing body of evidence from human and experimental data supports the role of lymphatics as an integral part of the intestinal immune-surveillance system by enabling efficient drainage to downstream lymph nodes of fluid, infiltrated immune cells, and antigen-presenting cells regulating immunity and subsequently promoting the resolution of inflammation.

An acute inflammatory response is the host's physiological and protective answer to invading pathogens, which is selflimited and ends with resolution of inflammation and ultimately tissue homeostasis. ${ }^{16}$ However, if these wellcontrolled resolution pathways fail, the acute inflammatory response becomes self-sustaining and chronic. Although the intestinal lymphatic vasculature forms the conduit to end antiinflammatory programs and enable resolution of inflammation, this dynamic process is driven by specialized endogenous proresolving mediators (SPMs). These include the $\operatorname{lipoxins}^{17,18}$ (LXs), resolvins ${ }^{19-21}$ (Rvs), protectins ${ }^{22}$ (PDs), and maresins ${ }^{23}$ (MaRs). The SPMs counterregulate the action of inflammation-initiating mediators, ${ }^{16,24}$ and their bioactive metabolomes have been shown to display several antiinflammatory-proresolving actions, including regulating proinflammatory mediators (eg, cytokines), dampening angiogenesis, accelerating clearance and efferocytosis of cells, and promoting epithelial barrier integrity. ${ }^{16,25}$

Resolution of inflammation is particularly important in the gut, where the mucosal surface and its immune system are in constant contact with pathogens and in an ongoing state of basal inflammation. To maintain a balance between physiological inflammatory responses and overwhelming destructive chronic inflammatory states, a meticulously orchestrated intestinal resolution program is mandatory. It has now been demonstrated that IBD patients are characterized by an imbalance between proinflammatory agents and proresolving molecules within the mucosal immune system. Masoodi et $\mathrm{al}^{26}$ demonstrated an increase of various arachidonic acid (AA)-derived lipid mediators (LMs) in inflamed colonic biopsies compared with noninflamed regions in UC patients. In addition, LM profiles correlated with the degree of histologic inflammation. Pearl et $\mathrm{al}^{27}$ demonstrated that UC patients had increased AA and docosahexaenoic acid (DHA) as well as reduced eicosapentaenoic acid (EPA) mucosal concentrations compared with healthy controls. This also correlated with the severity of mucosal inflammation. In line with these findings, Ungaro et $\mathrm{al}^{28}$ found reduced levels of proresolving DHAderived epoxy metabolites in regions with active inflammation in UC patients. Interestingly, in UC patients under remission, they also found elevated (compared with active disease) levels of these SPMs, suggesting that active UC is accompanied by a failure to produce SPMs and that these effects contribute to the induction of remission of mucosal inflammation in UC. In addition, SPMs, such as the omega-3 polyunsaturated fatty acids (PUFAs) derived resolvin E1, resolvin D1, resolvin D2, and MaR1 as well as more recently both protectin D1n-3 n-3 docosapentaenoic acid (DPA) and resolvin D5n-3 DPA, have each independently been shown to be protective in IBD. ${ }^{19,24-32}$ SPMs interact with specific receptors to temper leukocyte reactivity, dampen inflammatory pain, and promote tissue repair and regeneration; ${ }^{16}$ however, the possible interplay of the lymphatics as a structural intermediary between SPMs and the resolution of intestinal inflammation remains unexplored. In addition, issues still persist with animal models of intestinal inflammation as these current models most closely resemble clinical and histopathologic features of acute colitis, rather than CD.

In the 1970s, Kalima et $\mathrm{al}^{33}$ described an animal model of intestinal inflammation based on regional lymphatic obstruction, which closely resembled anatomic and clinical features of CD in humans. Although lymphatic obstruction alone does not reproduce the entire spectrum of CD pathology, it reproduces striking inflammatory similarities and many clinical features, including submucosal edema, thickening of the bowel wall, aggregation of lymphocytes, formation of lymphocytic and granulomatous changes, and the development of enteroenteric and enterocutaneous 

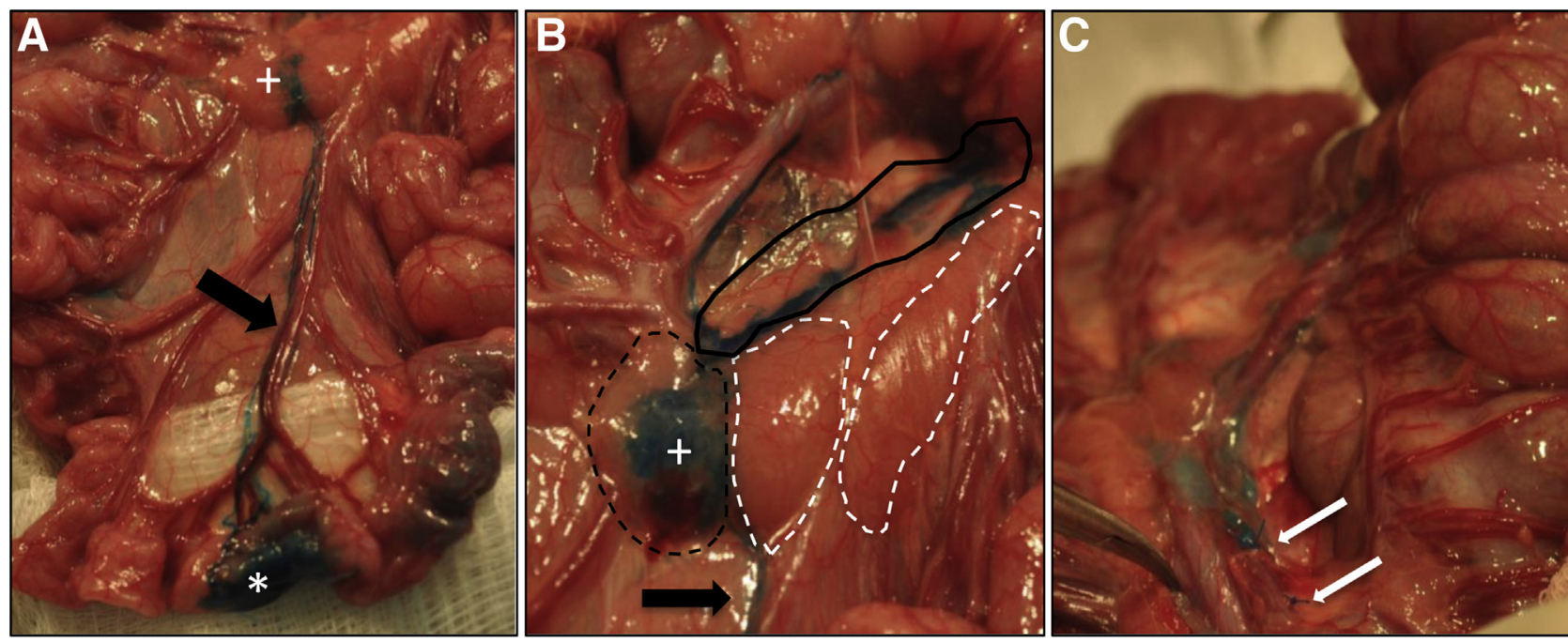

Figure 1 Identification of drainage lymph routes, lymph nodes, and surgically induced lymphatic obstruction (SLO). Representative images of SLO. A: Multiple ileal and cecal subserosal injections of isosulfan blue were conducted to specifically stain the lymphatic vessels and the respective draining lymph nodes. White asterisk marks the ileal subserosal isosulfan blue injection site. Black arrow indicates the draining mesenteric lymphatic vessels from the small intestine to the mesenteric lymph nodes, labeled with a white cross. B: Identification of the respective mesenteric lymph nodes after isosulfan blue injection to identify downstream lymph nodes (white cross) and adhered lymph node packages (dashed black line), as well as the drainage route to the cisterna chilly (solid black line). Lymph nodes from different ileal segments remain unstained (white dashed lines). C: The central lymphatic vessels at the base of the mesentery were ligated with nonreabsorbable sutures (6-0), avoiding manipulation of ileal/ileocolic arteries and veins. White arrows mark the respective ends after dissecting the central lymph vessel.

fistulae. ${ }^{33-35}$ To date, no available rodent animal model has ever shown such clinical similarities to CD and, in addition, there is no animal model currently available to study intestinal inflammation, which is based on lymphatic obstruction.

Herein, we recapitulated and further characterized the inflammatory model of regional lymphatic obstruction in nonhuman primates (NHPs), with a special focus on intestinal lymph flow, inflammatory markers, and mediators of resolution. A clinically relevant variation of the model of Kalima et $\mathrm{al}^{33}$ was recapitulated in NHPs with persistent blockade of regional lymph flow over a time course of 61 days. A severe inflammatory flare was observed, characterized by local and systemic up-regulation of inflammatory markers within the first 7 days, with a return to physiological homeostasis after 21 and 61 days, respectively. Using modern multipanel Luminex assays (Bio-Rad, Hercules, CA) and solid-phase extraction, coupled with LM-SPM liquid chromatography-tandem mass spectrometry (LC-MS/MS) - based metabololipidomics, smallbowel and colon samples were analyzed to identify unique signature profiles and relationship(s) between proinflammatory and anti-inflammatory cytokines, chemokines, and LMs and SPMs to assess their potential pathologic implications in the intestine of animals subjected to lymphatic blockade.

\section{Materials and Methods}

\section{Animals}

All animal protocols were approved by the University of Louisiana at Lafayette Animal Care and Use Committee and were handled in accordance with the American Association for Accreditation of Laboratory Animal Care guidelines.
Fifteen male African green monkeys (AGMs; Chlorocebus aethiops sabaeus; 2 to 4 years of age; weighing between 3.19 and $5.36 \mathrm{~kg}$ ) were used and maintained at the New Iberia Research Center, University of Louisiana at Lafayette.

Animals were socially housed during this study, except for a 1-week interval of separation postoperatively. Animals were fed daily with regular primate diet (5L2P*; Purina Lab, St. Louis, MO) in amounts appropriate for the size of the animals, which was supplemented with fruit, novel foodstuffs, and/or seed foraging at least five times as part of the New Iberia Research Center Plan for Environmental Enhancement and Behavioral Management. Tap water was provided ad libitum via automatic watering devices. Preoperatively, animals were fasted overnight with free access to water. Postoperatively, animals had free access to food and water ad libitum. Before the experiments, animals were screened for exclusion behavior (abnormal food/water consumption or stool consistency) to avoid an underlying gut-related wasting disease or infectious process. This included a physical examination by a veterinarian (E.R or D.L.H.), a complete blood cell count, a serum chemistry screen, and fecal testing for bacterial and parasitic pathogens. All animals were tested for SIV as well as simian T-cell leukemia virus type 1 and were found to be negative before inclusion in the experiments. Animals were randomized to either a treatment or a sham group.

\section{Experimental Groups}

To evaluate the course of intestinal injury after lymphatic obstruction, 15 animals were randomly assigned into three different experimental groups and one sham group. These 
groups were based on the observational period after the procedure: 7 (acute), 21 (midterm), or 61 (chronic) days. These time points were selected on the basis of earlier studies by Kalima et al, ${ }^{33,35}$ who reported lymphatic obstruction as a specific initiator of intestinal disease affecting multiple clinical parameters, which intensified over time. Each experimental group consisted of four animals. In addition, three animals were used as a control (sham), with one animal being sacrificed at 7, 21, and 61 days.

\section{Surgically Induced Lymphatic Obstruction}

Animals were sedated by i.m. injection of $10 \mathrm{mg} / \mathrm{kg}$ ketamine and $0.03 \mathrm{mg} / \mathrm{kg}$ buprenorphine, and a venous catheter was placed in a peripheral vein. Next, $5 \mathrm{mg} / \mathrm{kg}$ propofol was given intravenously, followed by endotracheal intubation. Anesthesia was maintained on isoflurane and propofol $(5 \mathrm{mg} / \mathrm{kg})$. Animals were placed in a supine position. The abdomen was shaved, and all the following surgical procedures were performed under sterile conditions. Briefly, a median laparotomy (approximately 3 inches) was performed, followed by an abdominal exploration to avoid congenital intra-abdominal findings. Next, the ileocecal region was located, and the last $20 \mathrm{~cm}$ of ileum and the first $10 \mathrm{~cm}$ of the cecum/ascending colon were measured and mobilized. The next step was to identify the lymphatic drainage route of the ileocecal region. Thus, multiple ileal and cecal subserosal injections of approximately $300 \mu \mathrm{L}$ isosulfan blue (1\%, Lymphazurin; Covidien, New Haven, CT) were administered to specifically stain the lymphatic vessels and the respective draining lymph nodes. This procedure rapidly $(<2$ minutes $)$ stained the mesenteric lymphatic vessels and later ( $>2$ minutes) the downstream proximal lymph nodes (Figure 1A). Next, 1\% isosulfan blue was injected in all stained lymph nodes to identify bigger downstream central lymph nodes at the mesenteric radix (Figure 1B). The so identified drainage route of the ileocecal region concordantly consisted regularly of one major central mesenteric lymph node, which represents the proximal downstream target for two to three ileal and three to five smaller colonic lymph nodes. Next, one to two central lymphatic vessels, which drained only from the major previously identified central mesenteric lymph node at the mesenteric radix into the cisterna chyli, were isolated. The proximal mesentery was then carefully opened, and the central lymphatic vessels at the base of the mesentery were ligated with nonreabsorbable sutures (6-0), avoiding manipulation of ileal/ileocolic arteries and veins (Figure 1C). To complete the lymphatic obstruction, 0.01 to $0.02 \mathrm{~mL}$ of $4 \%$ formalin (mixed with $1 \%$ isosulfan blue) was injected into the previously identified lymph nodes, again avoiding damage to lymph node capsules or manipulation of the ileal arteries and veins. Finally, the lymphatic obstruction was verified by reinjecting $1 \%$ isosulfan blue into the ileal and cecal subserosa. At this stage, a stasis of the blue dye in the mesenteric lymphatics and a complete reuptake in the proximal lymph nodes, without any leakage or outflow in the cisterna chyli, occurred. Afterward, the abdomen was closed in two layers using a continuous suture (muscle layer) and an interrupted suture technique (skin). Sham operations were performed under the exact same conditions, but only included opening of the abdominal wall, exploration, subserosal injection of $1 \%$ isosulfan blue, and closure of the abdominal wall. Postoperatively, no abdominal drain was placed and no postoperative antibiotics or nonsteroidal anti-inflammatory drugs were given. The postoperative analgesic regimen included acetaminophen $(6 \mathrm{mg} / \mathrm{kg}$, oral suspension p.o.) and tramadol (5 mg/kg, p.o.).

\section{Postsurgical Follow-Up}

Animals were followed up for 7, 21, or 61 days, examined daily (general condition, species-specific behavioral abnormalities, stool consistency, and appetite) by a veterinarian (E.R or D.L.H.), and assessed for a 5-step disease score (0 indicates very well; 1 , slightly below average; 2 , poor; 3 , very poor; or 4 , terrible). To conduct weekly examinations and blood collection, animals were sedated (ketamine, 10 $\mathrm{mg} / \mathrm{kg}$, intramuscularly) weekly until the time of euthanasia. Weekly assessments included body weight, temperature, heart rate, blood collection, as well as an abdominal examination by a veterinarian (E.R or D.L.H.).

\section{Necropsy}

At the designated study end point (7, 21, or 61 days), animals were fasted overnight before necropsy and at the day of euthanasia, sedated (ketamine, $10 \mathrm{mg} / \mathrm{kg}$, intramuscularly), followed by propofol induction and intubation. Anesthesia was maintained on isoflurane and propofol $(5 \mathrm{mg} / \mathrm{kg}$ ), if necessary. A blood sample was taken, the abdominal wall was opened, and the intra-abdominal conditions were examined. To control for successful surgically induced lymphatic obstruction (SLO), a dual approach and combined dye-based optical confirmation with conventional lymphography were used. Briefly, $5 \mathrm{~mL}$ of the radiographic contrast agent (Lipiodol; Guerbet, Bloomington, IN) was mixed with $300 \mu \mathrm{L}$ isosulfan blue. Next, $300 \mu \mathrm{L}$ of this solution was slowly injected into the prior sclerosed lymph nodes; and conventional $\mathrm{X}$-ray radiographs were taken in series after 5, 15, 25, and 30 minutes (Figure 2, A, E, and F). Radiographs were taken with a portable X-ray system (model HF100/30; MinXray, Inc., Northbrook, IL) and developed with a Fuji Prima T2 CR (Fujifilm, Valhalla, $\mathrm{NY}$ ). The addition of the lymph-specific dye isosulfan blue allowed a visual confirmation of the lymphostasis as well as irregular lymph flow (Figure 2, B-D). Euthanasia was performed under deep anesthesia using pentobarbital (120 $\mathrm{mg} / \mathrm{kg}$ i.v.) injection, followed by cardiac puncture/pneumothorax. After euthanasia, the entire ileocecal region was removed, opened longitudinally, washed with cold phosphate-buffered saline, and macroscopically assessed; subsequently, tissue samples were harvested. 




\section{Blood Analysis}

Blood samples were collected 1 week before the study, at the day of surgery, and weekly until euthanasia. Blood analysis was conducted at the University of Louisiana at Lafayette-New Iberia Research Center Clinical Pathology Laboratory, including comprehensive serum chemistry panel with lipids (no anticoagulant) screen (Clinical Chemistry Analyzer; Siemens, Munich, Germany); a hematology panel (EDTA tubes) with complete blood cell count, differential, and platelet count (LH780; Beckman Coulter, Brea, CA); and coagulation profile (ACL Elite Coagulation Analyzer; Instrumentation Laboratory, Bedford, MA), including prothrombin time, activated partial thromboplastin time, and fibrinogen analysis (sodium citrate tubes).

\section{Histology}

Tissue samples of the ileocecal region (ileum and colon) as well as mesenteric lymph nodes were first fixed in $10 \%$ phosphate-buffered formalin, then transferred to $70 \%$ ethanol, embedded in paraffin, divided into sections $(5 \mathrm{~mm}$ thick), and subsequently stained with hematoxylin and eosin. Histologic examination was conducted by a boardcertified and specialized veterinary pathologist (D.L.H.).

\section{Luminex Analysis}

Local tissue (ileocecal region) as well as systemic (serum) levels of inflammatory markers were assed using Luminex fluorescent bead human cytokine and chemokine immunoassay kits (Bio-Rad), according to the manufacturer's instructions. Serum samples were collected from each animal into vacutainer tubes with no anticoagulant at the time of prestudy physical examinations, the day of surgery, and weekly until euthanasia. Samples were maintained at room temperature until clot formation, centrifuged at $1000 \times g$ for 15 minutes at $4{ }^{\circ} \mathrm{C}$, aliquoted, and subsequently stored at $-70^{\circ} \mathrm{C}$. Samples collected after 7,21 , or 61 days were compared with prestudy values as a control group. Tissues samples of the ileocecal region (colon and ileum) were snap frozen, and lysates were prepared using a Bio-Plex cell lysis kit (Bio-Rad), according to the manufacturer's instructions. Lysates were diluted in sample diluent $+0.5 \%$ bovine serum albumin (Sigma-Aldrich, St. Louis, MO), to a final protein concentration of $300 \mu \mathrm{g} / \mathrm{mL}$. Samples were analyzed on a Luminex MAGPIX (Bio-Rad) at the New Iberia Research Center-University of Louisiana at Lafayette.

\section{Targeted Lipid Mediator-SPM Metabololipidomics}

Tissue extraction and LM metabololipidomics were conducted as described previously. ${ }^{36}$ Briefly, LC-grade solvents (Fisher Scientific, Pittsburgh, PA), C18 solid-phase extraction columns (500 mg; Biotage, Uppsala, Sweden), Poroshell 120 EC-C18 2.7- $\mu \mathrm{m}$ column $(100 \times 4.6 \mathrm{~mm}$; Agilent, Santa Clara, CA $)$, and synthetic standards and deuterated internal standards (Cayman Chemical, Ann Arbor, MI) were used. Briefly, colon and small-bowel samples were defrosted from $-80^{\circ} \mathrm{C}$ on ice. Tissues were weighed and gently dispersed with a glass Dounce. Internal labeled standards $\mathrm{d}_{8}-5 S$-HETE (5S-hydroxy6E,8Z,11Z,14Z-eicosatetraenoic-5,6,8,9,11,12,14,15-d8 acid), $\mathrm{d}_{4}$ $\mathrm{LTB}_{4}$ (5S,12R-dihydroxy-6Z,8E,10E,14Z-eicosatetraenoic6,7,14,15-d4 acid), $\mathrm{d}_{5}-\mathrm{LXA}_{4}$ (5S,6R,15S-trihydroxyicosa7E,9E,11Z,13E-tetraenoic-19,19,20,20,20-d5 acid), $\mathrm{d}_{5^{-}}$ RvD2 (resolvin D2), and $\mathrm{d}_{4}$-prostaglandin $\mathrm{E}_{2}\left(\mathrm{PGE}_{2}\right)(500$ $\mathrm{pg}$ each) in $4 \mathrm{~mL}$ of ice-cold methanol were added to each sample to facilitate quantification and sample recovery. Next, samples were held at $-20^{\circ} \mathrm{C}$ for 45 minutes to allow protein precipitation and then centrifuged $\left(1200 \times g, 4^{\circ} \mathrm{C}, 10 \mathrm{mi}-\right.$ nutes). Supernatants were collected and brought to $<1 \mathrm{~mL}$ of methanol content in a gentle stream of nitrogen gas onto an automated evaporation system (TurboVap LV; Biotage, Charlotte, NC). Samples were then placed into an automated extraction system (Extrahera; Biotage, Charlotte, NC), and products were extracted, as previously described ${ }^{37}$ and as described further. Briefly, solid-phase $\mathrm{C} 18$ cartridges were equilibrated with $3 \mathrm{~mL}$ of methanol and $3 \mathrm{~mL}$ of $\mathrm{H}_{2} \mathrm{O}$. A total of $9 \mathrm{~mL}$ of $\mathrm{H}_{2} \mathrm{O}$ ( $\mathrm{pH} 3.5$, hydrochloric acid) was added to the samples, and the acidified solutions were rapidly loaded onto the conditioned $\mathrm{C} 18$ columns that were washed with $3 \mathrm{~mL}$ of $\mathrm{H}_{2} \mathrm{O}$ to neutralize the acid. Next, $3 \mathrm{~mL}$ of hexane was added, and products were eluted with $3 \mathrm{~mL}$ of methyl formate. Products were brought to dryness using the automated evaporation system (TurboVap LV; Biotage, Charlotte, NC) and immediately suspended in methanol-water $(50: 50 \mathrm{v} / \mathrm{v})$ for LC-MS/MS automated injections.

\footnotetext{
Figure 2 Persistent lymphostasis coupled with lymph backflow induced by surgically induced lymphatic obstruction (SL0). After the respective follow-up period of 7, 21, or 61 days, all animals (including sham) underwent a relaparotomy for evaluation of SL0 by intestinal lymphography. A: Representative conventional X-ray radiograph, taken after the injection of a lymph-specific dye [isosulfan blue (Lymphazurin)] and a radiographic contrast agent (Lipiodol) into mesenteric lymph nodes, shows the regular outflow from the intestine via intestinal lymphatics into the cisterna chyli (white arrow) and into the thoracic duct in sham animals. Representative images taken from SLO animals after injection of isosulfan blue into prior sclerosed lymph nodes. B and C: Images taken 7 (B) and 21 (C) days after SLO. White asterisks indicate the injection side; black dashed lines, the pooling of dye without any outflow from the respective lymph node. The mesenterium appears thickened with a fibrin layer at 7 days (B) and looks recovered, but signs of lymphostasis remain at 21 days (C). D: Image taken 21 days after SLO. White arrows show irregular backflow of dye from the lymph node into the small bowel. These findings were confirmed by intestinal lymphography showing pooling of contrast agent in the sclerosed lymph nodes without any clearance into the cisterna chyli. $\mathbf{E}$ and $\mathbf{F}$ : Data for 7 (E) or 61 (F) days after SLO. White arrows show the regular outflow from the intestine via intestinal lymphatics into the cisterna chyli. G: Hematoxylin and eosin (H\&E) section of sclerosed intestinal lymph nodes reveals necrotic zones without any evidence of further damage to the capsule and shows a rich immune cell infiltration. H and I: H\&E-stained cross-sections were examined for signs of inflammation and lymphostasis. H: Pronounced serosal reaction and vasculitis (black asterisk). I: Profound lacteal dilation (black crosses) in the villar tips as well as a serosal lymphatic dilation.
} 
A

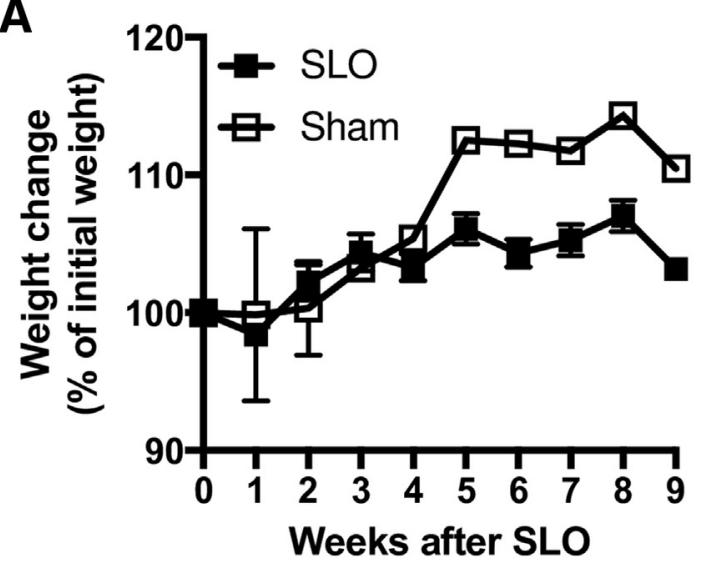

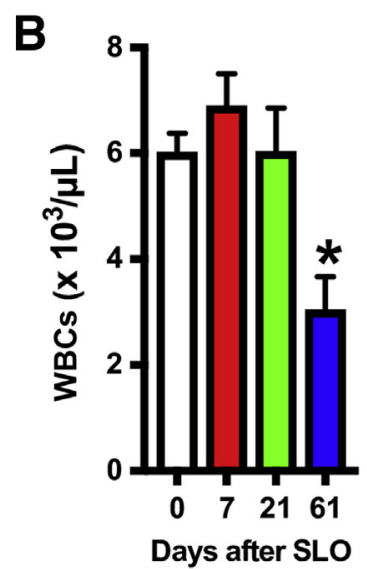

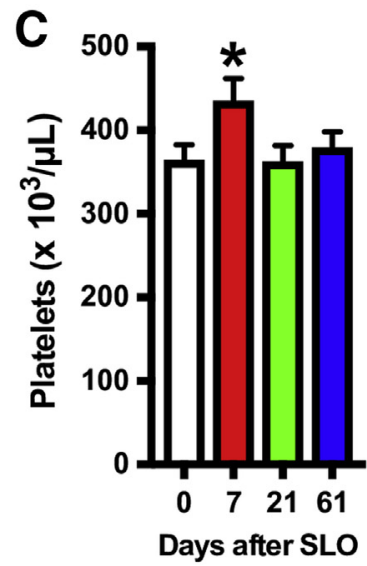

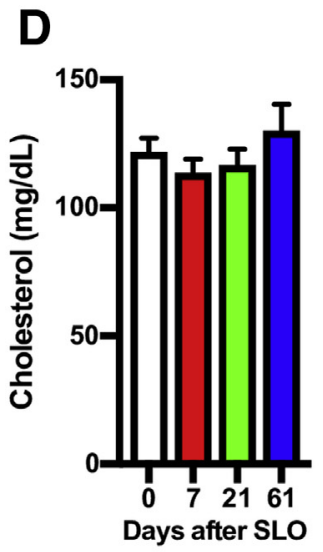


H



Figure 3 Markers of disease progression after surgically induced lymphatic obstruction (SLO). Sham animals (0 days after SL0) and animals undergoing SLO were subjected to the weekly clinical examination and blood testing up to the respective time point of necropsy (7, 21, and 61 days). A: Compared with the sham group (open boxes), animals subjected to SLO (closed boxes) showed a slight decline in weight gain over the course of the experiment; however, it is not statistically significant. B: SLO results in a 12\% increase after 7 days in white blood cell counts (WBCs), as well as a significant decrease in WBCs at 61 days, compared with the sham group. C: SLO results in a significant increase in platelet count after 7 days, which returns to baseline values at 21 and 61 days after SLO. D-H: No differences are seen when analyzing the laboratory lipid profile consisting of cholesterol (D), direct low-density lipoprotein (LDL) cholesterol (E), high-density lipoprotein (HDL) cholesterol (F), triglyceride (G), and albumin (H). B-G: The control group (0 days after SL0) includes samples from all animals, taken at the prestudy examination. Data are representative of 15 prestudy control African green monkeys (AGMs) at 0 days after SLO, 12 experimental AGMs at 7 days after SLO, 8 AGMs at 21 days after SLO, and 4 AGMs at 61 days after SL0. Data are expressed as means \pm SEM $(\mathbf{A}-\mathbf{H})$. ${ }^{*} P<0.05$ versus sham.

\section{Statistical Analysis}

Statistical analysis was conducted with Graph Pad Prism software version 7 (GraphPad Software, San Diego, CA). Groups were compared using one-way analysis of variance, followed by Bonferroni post hoc testing. Data were expressed as average \pm SEM, and $P<0.05$ was considered statistically significant. All $n$ values are indicated in the respective figure legends.

\section{Results}

\section{AGMs Undergoing SLO Display No Signs of Morbidity}

All animals recovered quickly from surgery, irrespective of sham procedures or SLO. In addition, no differences were found with respect to the daily clinical disease score (data not shown). Compared with the sham group, animals subjected to
SLO showed a slight reduction in weight gain over the course of the experiment; however, this was not statistically significant (Figure 3A). When animals were subjected to the weekly clinical examination, the full-body examinations revealed no wound healing disorders. In addition, no animal developed fever, showed a significant increase in heart rate, or had diarrhea or steatorrhea. The weekly laboratory panel revealed a $12 \%$ increase after 7 days in white blood cell count, as well as a significant decrease in white blood cell count at 61 days (Figure 3B). In addition, intestinal inflammation after SLO induced a significant increase in platelet count after 7 days, which returned to baseline values at 21 and 61 days (Figure 3C). Because intestinal lymphatics are the conduit for dietary lipids, a laboratory lipid profile, consisting of cholesterol (Figure 3D), direct low-density lipoprotein cholesterol (Figure 3E), high-density lipoprotein cholesterol (Figure 3F), triglyceride (Figure 3G), and albumin (Figure $3 \mathrm{H}$ ), was tested on a weekly basis, to assay for any 

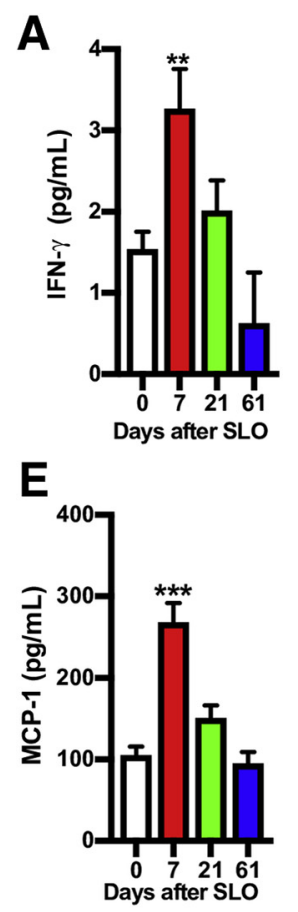

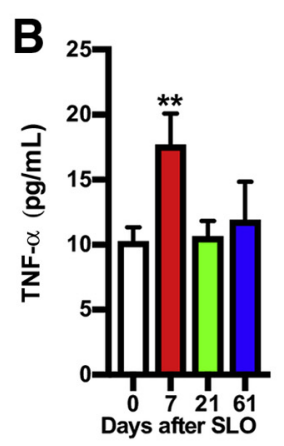

$\mathbf{F}$

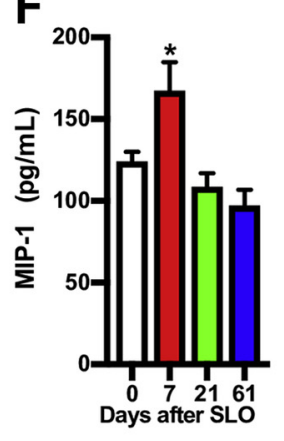

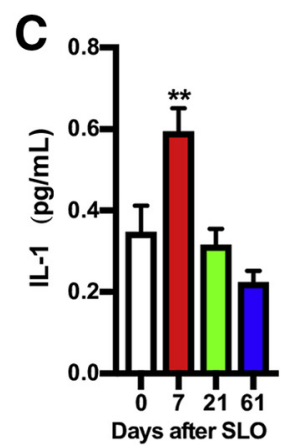

G

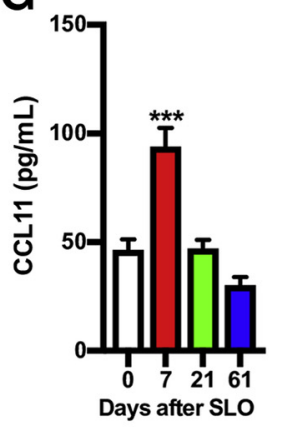

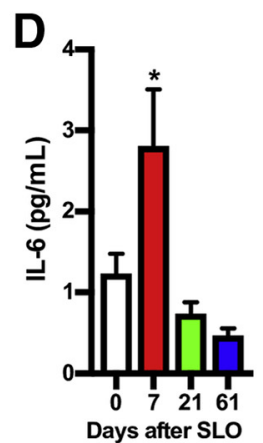

$\mathbf{H}$



\section{Serum}
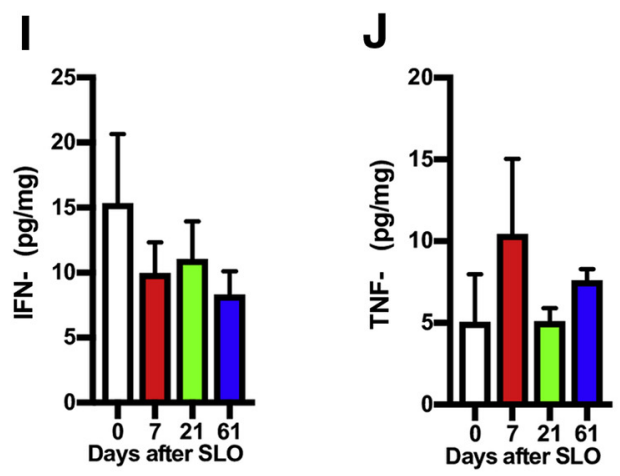

K


\section{$\mathbf{L}$}


Tissue

Figure 4 Local and systemic chemokine and cytokine profiles after surgically induced lymphatic obstruction (SLO). A-H: Serum samples were collected from experimental animals at 7, 21, or 61 days after SLO. The control group (0 days after SL0) includes samples from all animals, taken at the prestudy examination. A-H: Data are representative of 15 prestudy control African green monkeys (AGMs) at 0 days after SL0, 12 experimental AGMs at 7 days after SL0, 8 AGMs at 21 days after SLO, and 4 AGMs at 61 days after SL0. I-P: Tissue samples (ileum) were harvested at 7, 21, and 61 days after SLO. Samples from sham animals (0 days after SL0) were also taken at these time points. All serum samples were analyzed for changes in chemokines and cytokines using Luminex immunoassay kits. I-P: Data are representative of four AGMs with SLO per time point and one sham per time point. Data are expressed as means $\pm \mathrm{SEM}$. ${ }^{*} P<0.05,{ }^{* *} P<0.01$, and ${ }^{* * *} P<0.001$ versus sham. CCL, chemokine (C-C motif) ligand; IFN- $\gamma$, interferon- $\gamma$; MCP-1, monocyte chemoattractant protein-1; MIP-1, macrophage inflammatory protein-1; TNF- $\alpha$, tumor necrosis factor- $\alpha$. 

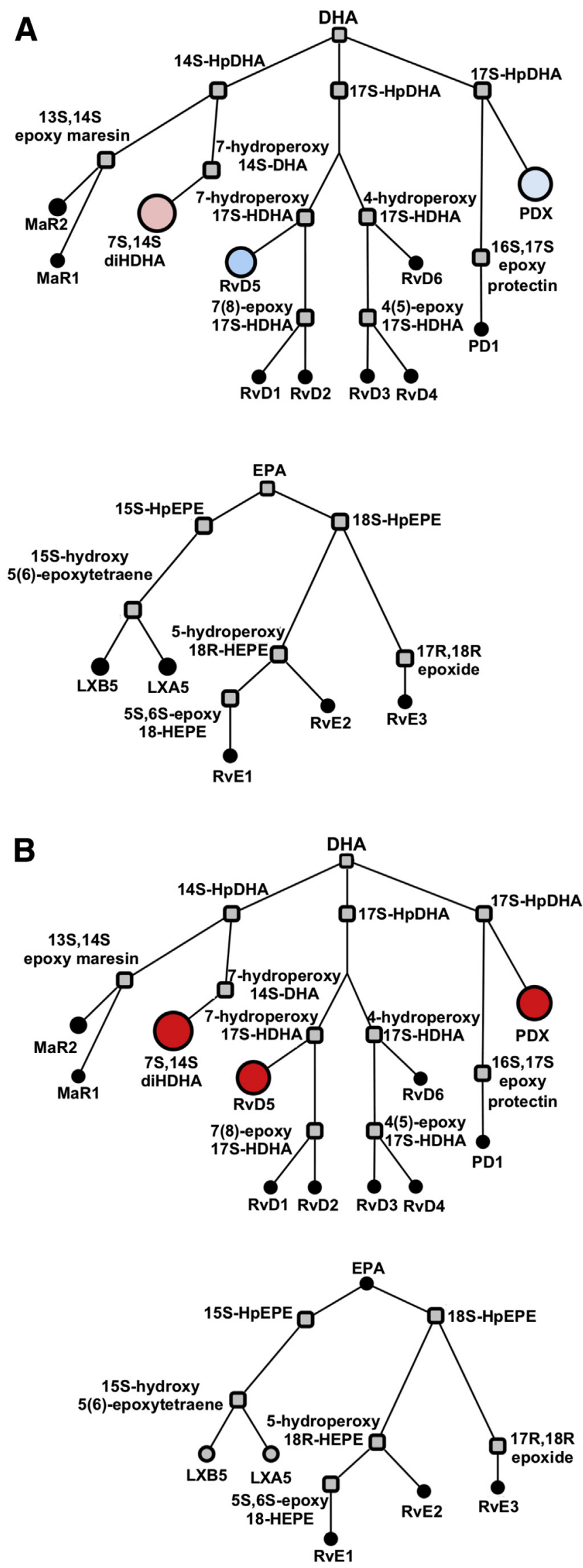
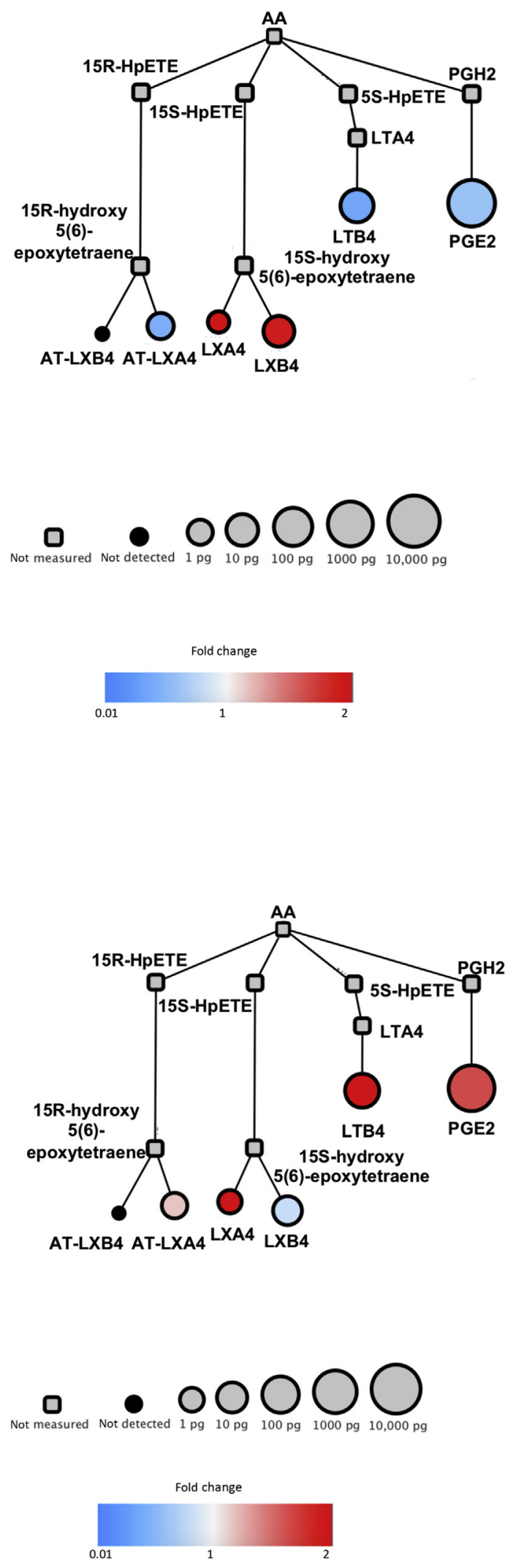
systemic changes in the lipid profile of the experimental animals secondary to SLO. However, SLO induced no systemic changes in the above mentioned parameters.

\section{AGMs Exhibit Macroscopic Findings of a Regional Ileocolic Inflammation within 7 Days after SLO}

After the respective follow-up period of 7, 21, or 61 days, all animals (including sham) underwent a relaparotomy for sample collection and evaluation of SLO. Animals in the 7-day group presented with several intra-abdominal alterations. The mesentery in the ileocecal region was thickened, shortened, and covered with a fibrin layer, whereas the bowel loops appeared edematous. The prior sclerosed lymph nodes were hyperplastic and hardened. In addition, adhesions between different loops of the small bowel and the mesentery occurred. The findings were absent in all sham animal as well as in the 21- and 61-day experimental animal group.

\section{SL0 Results in Persistent Lymphostasis Coupled with Lymph Backflow}

A dual approach was used to confirm successful SLO in the experimental animals and demonstrate an undisturbed intestinal lymphatic outflow in sham animals. An oil-based contrast agent was mixed with a lymph-specific dye (isosulfan blue) and injected into the prior sclerosed lymph nodes. Sham animals showed a normal lymph outflow, without any signs of dye pooling. Next, these findings were verified using conventional X-rays (lymphography). Sham animals presented with an undisturbed lymph clearance via the cisterna chyli into the central circulation (Figure 2A). By comparison, animals after SLO presented with an initial pooling of the blue dye in the prior sclerosed lymph nodes due to the obstructed lymph efflux as well as a nonphysiological retrograde lymph into the bowel wall (Figure 2, B-D). When conventional lymphography was used to further visualize the lymph outflow, experimental animals showed complete pooling of the injected contrast agent in the mesenteric lymph nodes and no outflow into the cisterna chyli (Figure 2, E and F). These findings were consistent in all experimental groups, irrespective of observational time.

\section{AGMs Presented with Intestinal Lacteal Dilation within 7 Days of SLO}

To further validate the local effects of SLO on the intestinal architecture, hematoxylin and eosin-stained sections of sclerosed lymph nodes, as well as colon and small-bowel samples, were analyzed. Although no changes were observed in sham animals, lymph nodes in the experimental groups were partially necrotic without any evidence of further damage to the capsule and showed a rich immune cell infiltration (Figure 2G). In tissue samples from the ileocecal region serosal reaction, vasculitis and an increase in lamina propria/submucosal infiltration of immune cells, such as macrophages, were observed (Figure $2 \mathrm{H}$ ). These changes were most prominent in the 7-day animals and subsided at later time points. In addition, a profound lacteal dilation was found in the villar tips as well as a serosal lymphatic dilation (Figure 2I), confirming the result of lymphostasis and disrupted lymph flow from the intestine to the respective lymph nodes.

\section{SLO Leads to a Temporal Regulation in Systemic Inflammation in AGMs}

Serum samples (collected before and 7, 21, or 61 days after SLO) were analyzed for changes in chemokines and cytokines using Luminex immunoassay kits. Three critical proinflammatory mediators [interferon- $\gamma$ (Figure 4A), tumor necrosis factor- $\alpha$ (TNF- $\alpha$ ) (Figure 4B), and IL-1 $\beta$ (Figure 4C)] were found to be up-regulated 7 days after SLO but returned to baseline levels after 21 or 61 days. The analysis was further expanded, and the concentrations of IL-1ra, IL-2, IL-4, IL-5, IL-6, IL-7, IL-8, IL-9, IL-10, IL-12, IL-13, and IL-16 were measured. Concentrations of IL-1ra, IL-6 (Figure 4D), IL-8, IL-12, IL-13, and IL-16 were increased after 7 days and returned to baseline levels after 21 or 61 days (data not shown). Next, serum chemokine (C-C motif) ligand (CCL) chemokines [CCL-1, monocyte chemoattractant protein-1 (MCP-1)/CCL2, CCL-3, macrophage inflammatory protein-13/CCL4, CCL-5, CCL-8, CCL-11, CCL-13, CCL-15, CCL-19, CCL-20, CCL-21, CCL-22, CCL-25, and CCL-27], CXCL chemokines (CXCL1, CXCL2, CXCL8, CXCL9, CXCL10, CXCL11, and CXCL13), and chemokine (C-X3-C motif) ligand 1 (CX3CL1, also known as fractalkine) were measured. Significant increases were observed 7 days after SLO for MCP-1 (Figure 4E), macrophage inflammatory protein-1 $\beta$ (Figure 4F), CCL11 (Figure 4G), and CCL15 (Figure 4H) as well as for CCL-5, CCL-8, CCL-21, CCL-22, CXCL9, and CXCL11 (data not shown). These increased levels all returned to baseline levels after 21 and 61 days. Only CX3CL1 was significantly reduced 7 days after SLO.

\section{AGMs Display a Temporal Regulation in Local Inflammation after SLO}

Having determined the effects on systemic inflammatory markers after SLO, changes were studied at a local level. As

\footnotetext{
Figure 5 Specialized proresolving lipid mediator biosynthesis in the small bowel of African green monkeys (AGMs). Ileum samples were taken from AGMs at 7,21 , and 61 days after surgically induced lymphatic obstruction (SLO). Samples from sham animals (0 days after SLO) were also taken at these time points. Lipid mediators (LMs) were assessed using targeted liquid chromatography-tandem mass spectrometry (LC-MS/MS)-based LM metabololipidomics (Materials and Methods). Cytoscape network analysis shows changes in the docosahexaenoic acid (DHA), arachidonic acid (AA), and eicosapentaenoic acid (EPA) metabolomes at 21 versus 7 days (A) and 61 versus 21 days (B) after SLO. Colors represent the magnitude of fold change (red indicates increased; blue, decreased), and the size of the circle correlates with abundance ( $\mathrm{pg} / 500 \mathrm{mg}$ tissue). Data are expressed as means. $n=4$ AGMs with SL0 per group.
} 

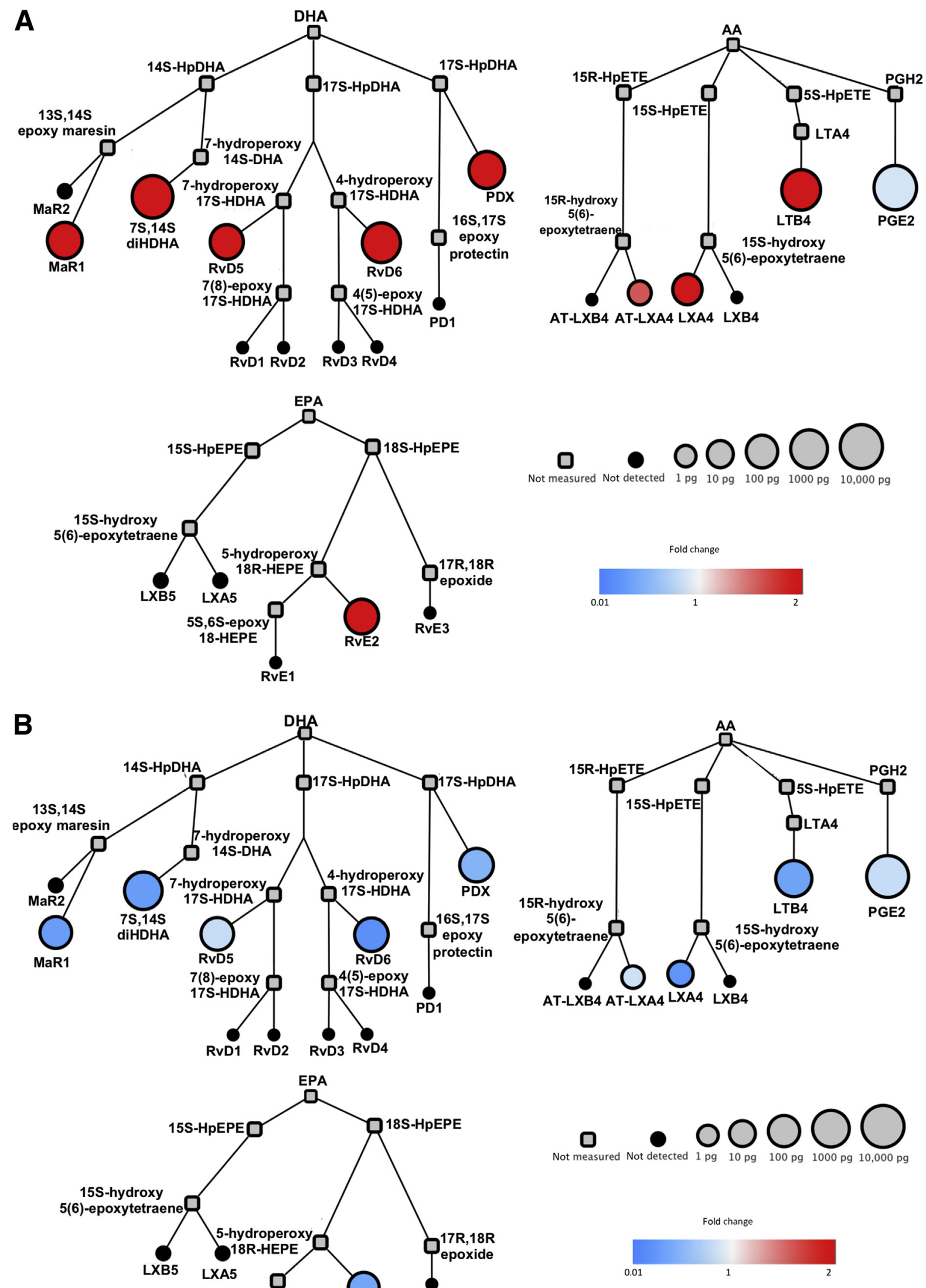
such, tissue samples of the ileocecal region (colon and terminal ileum) were analyzed for the presence of inflammatory markers. Unlike serum markers, the inflammatory profile in the ileocecal region was found to be variant. In general, a similar trend to that seen with systemic inflammation after SLO (ie, an up-regulation of inflammatory chemokines and cytokines 7 days after SLO) was observed, which returned to physiological baseline at 21 and 61 days. Although MCP-1 (Figure 4M) and CCL11 (Figure 4O) were significantly increased, TNF- $\alpha$ (Figure 4J), IL-6 (Figure 4L), macrophage inflammatory protein-1 $\beta$ (Figure 4N), and CCL15 (Figure 4P) showed a trend toward an increase after 7 days, but this did not reach statistical significance. No differences were noted for interferon- $\gamma$ (Figure 4I) and IL-1 $\beta$ (Figure 4K).

\section{AGMs Display a Distinct Profile of SPMs}

Using targeted LC-MS/MS-based LM metabololipidomics, both proinflammatory and anti-inflammatory LMs and SPMs were profiled from AA-, EPA-, and DHA-derived bioactive metabolomes in the ileocecal region of sham animals and animals subjected to SLO. The presence of classic proinflammatory AA-derived eicosanoids, such as prostaglandins and leukotrienes, was also determined. In sham AGMs and AGMs subjected to SLO, members of all five main families of SPMs (AA-derived lipoxins, EPA-derived resolvins, DHA-derived resolvins, protectins, and maresins) were identified (Figures 5 and 6 and Supplemental Tables S1 and S2). Related biosynthetic isomers were also identified for select SPMs, including 10S,17S-diHDHA (a protectin biosynthetic isomer), 7S,14S-diHDHA (a maresin biosynthetic isomer), and 5S,15S-diHETE (a lipoxin biosynthetic isomer), each produced by double lipoxygenation reactions (Figures 5 and 6). These were identified in accordance with published criteria that included matching retention times, fragmentation patterns, and at least six characteristic and diagnostic ions for each. ${ }^{36}$

\section{SPMs Are Up-Regulated in the Small Bowel 61 Days after SLO}

Using LC-MS/MS-based LM metabololipidomics to analyze LMs in the terminal ileum of AGMs after sham or SLO, nine bioactive mediators and pathway products were found from DHA, AA, and EPA bioactive metabolomes (Figure 5). As shown by respective multiple reaction monitoring chromatograms of selected ion pairs and representative MS/MS fragmentation spectra used for identification (Supplemental Figure S1), RvD5, the protectin pathway markers 10S,17S-diHDHA (PDX), and 7S,14SdiHDHA, $\mathrm{LXA}_{4}, \mathrm{LXB}_{4}$, aspirin-triggered (AT) $\mathrm{LXA}_{4}$, 5S,15S-diHETE, and leukotriene B4 $\left(\mathrm{LTB}_{4}\right)$, as well as $\mathrm{PGE}_{2}$ were identified. The identified proinflammatory and anti-inflammatory LMs were further quantified and expressed in a Cytoscape network analysis (Figure 5), showing changes in the DHA, and AA metabolomes at 21 versus 7 days and at 61 versus 21 days after SLO. After the acute inflammatory state at 7 days after SLO, an increase was found in the AA-derived proresolving mediators $\mathrm{LXA}_{4}$ and $\mathrm{LXB}_{4}$ as well as DHA-derived 7S,14S-diHDHA, with a decrease of AA-derived proinflammatory $\mathrm{LM} \mathrm{PGE}_{2}$ and $\mathrm{LTB}_{4}$, suggesting that SPMs are produced in the small bowel of AGMs after SLO and can undergo a proinflammatory class switch after day $7 .{ }^{16}$ When 61-day animals were compared with animals 21 days after SLO, a further increase was noted in DHA-derived 7S,14S-diHDHA, RvD5, and 10S,17S-diHDHA (Figure 5).

Next, LMs were combined into groups to compare cumulative levels for D-series resolvins, protectins, and maresins, lipoxins, and prostaglandins and leukotrienes (Supplemental Figure S1). Interestingly, a significant increase for D-series resolvins, protectins, and maresins at 61 days after SLO was found, compared with 7-day animals. A similar trend was observed for lipoxins, although statistically significant changes in proinflammatory (leukotriene and prostaglandin) LM levels were not observed. These data demonstrate, for the first time, distinct changes in the SPM profiles of AGMs in relation to a model of intestinal inflammation.

\section{SPMs Are Produced in the Colon of AGMs}

The targeted LC-MS/MS-based LM metabololipidomics approach was used to profile AA, EPA, and DHA bioactive metabolomes in colon tissue from AGMs after sham or SLO. Eleven bioactive mediators and pathway products were identified from AA, DHA, and EPA bioactive metabolomes (Figure 6). As shown by respective multiple reaction monitoring chromatograms of selected ion pairs and representative MS/MS fragmentation spectra used for identification (Supplemental Figure S2), DHA-derived MaR1, 7S,14S-diHDHA, RvD5, RvD6, and PDX, AA-derived AT LXA 4 , 5S,15S-diHETE, $\mathrm{LXA}_{4}, \mathrm{LTB}_{4}$, as well as $\mathrm{PGE}_{2}$ and EPA-derived RvE2 were identified. The identified proinflammatory and anti-inflammatory LMs were again quantified and expressed in a Cytoscape network analysis (Figure 6), showing changes in the DHA, AA, and EPA metabolomes in the colon at 21 versus 7 days and at 61

\footnotetext{
Figure 6 Specialized proresolving lipid mediator biosynthesis in the colon of African green monkeys (AGMs). Colon samples were taken from AGMs 7, 21, and 61 days after surgically induced lymphatic obstruction (SLO). Lipid mediators (LMs) were assessed using targeted liquid chromatography-tandem mass spectrometry (LC-MS/MS)-based LM metabololipidomics (Materials and Methods). Cytoscape network analysis shows changes in the docosahexaenoic acid (DHA), arachidonic acid (AA), and eicosapentaenoic acid (EPA) metabolomes at 21 versus 7 days (A) and 61 versus 21 days (B) after SL0. Colors represent the magnitude of fold change (red indicates increased; blue, decreased), and the size of the circle correlates with abundance (pg/500 mg tissue). Data are expressed as means. $n=4$ AGMs with SLO per group.
} 


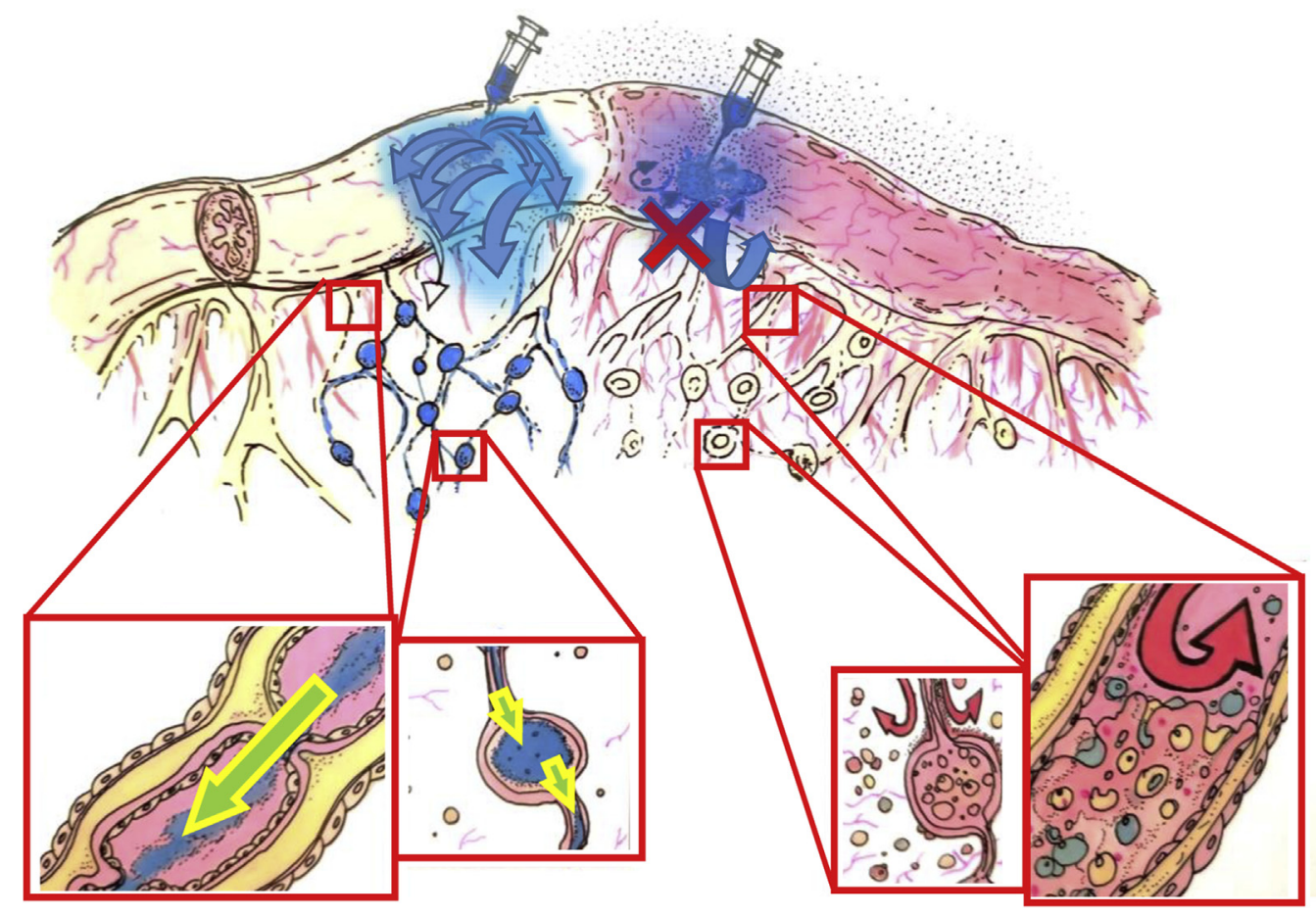

Figure 7 Schematic overview of surgically induced lymphatic obstruction (SLO). Left panels: Normal lymphatic vessel structure and function in sham African green monkeys (AGMs): Subserosal injection of the lymph-specific dye [isosulfan blue (Lymphazurin)] rapidly stains lymphatic capillaries within the bowel wall with a unidirectional flow (yellow/green arrows) into collecting vessels within the mesentery without any signs of dye pooling. Right panels: Disturbed lymphatic structure and function in AGMs after SLO: Subserosal dye injection leads to lymphostasis within the lymphatic capillaries of the bowel wall. The mesentery in the ileocecal region is thickened and shortened, whereas the bowel loops are edematous. The prior sclerosed lymph nodes show no sign of physiological lymph transport (black/red arrows).

versus 21 days after SLO. When animals 21 days after SLO were compared with animals 7 days after SLO, an increase in 9 of the 10 (MaR1, 7S,14S-diHDHA, RvD5, RvD6, PDX, AT LXA 4 , LXA 4 , LTB 4 , and RvE2) identified bioactive metabolomes in colon tissue was seen, with a slight decrease in $\mathrm{PGE}_{2}$ (Figure 6). When animals 61 days after SLO were compared with animals 21 days after SLO, global reduction was seen in all detected SPM and proinflammatory mediators (Figure 6). The cumulative LM profiles for D-series resolvins, protectins, and maresins (Supplemental Figure S2C), E-series resolvins (Supplemental Figure S2D), lipoxins (Supplemental Figure S2E), and prostaglandins and leukotrienes (Supplemental Figure S2F) were next assessed. There was an apparent trend toward a decrease in LM production at day 7, which was followed by an increase at day 21 after SLO. These results demonstrate that SPMs are produced in AGM colon tissue and are temporally regulated after SLO.

\section{Discussion}

The current hypothesis on the pathogenesis of IBD is that a dysregulated intestinal microbiome initiates an inappropriate inflammatory response by the genetically susceptible host's mucosal immunity, leading to chronic intestinal inflammation. Although targeting inflammatory pathways has not resulted in sufficient and steady relief from IBD, recent data strongly indicate that a failure in resolving inflammation might also contribute to the pathogenesis of this disease.$^{38}$ In addition, a growing body of evidence suggests a pivotal role of lymphatics in IBD's pathogenesis; however, the distinct changes in the intestinal lymphatic vasculature, which could be a cause or consequence of the inflammatory state, are only understood insufficiently. ${ }^{2}$ In the present study, an inflammatory model of regional lymphatic obstruction was recapitulated and renewed in NHPs (Figure 7). Systemic and local inflammatory profiles were analyzed. Identification of endogenous SPMs in AGMs was documented for the first time, and LM-SPM signatures of serum and ileocecal tissue in AGMs after SLO were described. These results show an acute inflammatory response in AGMs to SLO, followed by a phase of resolution of inflammation, accompanied by both an increase and a decrease of respective proinflammatory cytokines and chemokines as well as SPMs.

Lymphatic vessels have traditionally been considered as the principal conduit for a passive efferent removal of interstitial fluid, but new functions and underlying molecular mechanisms are emerging. ${ }^{3,4}$

Among the eminent but yet unanswered questions within the lymphatic research community is whether lymphatic changes are consequences or causes of the inflammatory state seen in human IBD. As for CD, there are early reports describing its pathologic pattern as a chronic lymphocytic, 
granulomatous lymphangitis, suggesting a primary role for lymphatics in the initiation and perpetuation of this disease. On the other hand, there is clear evidence that intestinal inflammation itself elicits adaptive changes in function and formation of lymphatic networks and that $\mathrm{CD}$ is characterized by a process of transmural lymphangiogenesis in the ileum and colon, ${ }^{7,8}$ accompanied by an up-regulation of prolymphangiogenic factors, such as vascular endothelial growth factor-C, podoplanin, or lymphatic vessel endothelial hyaluronan receptor 1 (LYVE-1) ${ }^{13}$ On the other hand, there are clear signs of lymphatic transport failure in $\mathrm{CD}{ }^{5}$ This generates an obvious conflict between an expanded, but yet apparently insufficient, intestinal lymphatic network in IBD. Among the possible explanations for this is a diminished drainage function, based on impaired fluid, antigen and immune cell uptake, ${ }^{10}$ lymphatic obstruction, ${ }^{39}$ or compromised lymphatic contractile function. ${ }^{40}$ This might promote lymphatic stasis, lymphangitis, and fluid as well antigen accumulation, all of which subsequently leads to an inflammatory state. However, if these are lymphangitispreceding events or caused by an initial damage to the lymphatic vasculature remains unknown. Irrespective of the sequence of these events, promoting endogenous resolution has the potential to improve lymphatic function because it was demonstrated that enhanced resolution reduced concentrations of inflammatory mediators, such as MCP-1, IL6 , IL-8, IL-1 $\beta$, and cyclooxygenase 2 in a colitis model. ${ }^{28}$ This is of interest because especially the last two mediators have been shown to directly alter lymphatic vessel contractility and hence lymph flow. ${ }^{40,41}$

It was shown that expansion of intestinal lymphatics reduced intestinal inflammation ${ }^{12}$ and disturbances in efferent lymph flow $^{15}$ or reduced lymphatic expansion-increased intestinal inflammation. ${ }^{11}$ On the basis of these results, one could hypothesize that disturbances of intestinal lymph flow cause alterations, which ultimately lead to intestinal inflammation; this is what Kalima $^{33}$ and Kalima and Collan ${ }^{34}$ described in 1970 when first reporting on their results from experimental lymphatic obstruction in rats. In the present study, the intra-abdominal findings after SLO are in line with observations by Kalima et al, ${ }^{33,34}$ who also reported adhesions with fibrin layers, a thickened mesentery, and edematous bowel loops. Another feature described by Kalima et $\mathrm{al}^{33,34}$ and present in this study was signs of lymphatic transport failure evident in serosal edema, accompanied by distinct dilatation of the lacteals within the mucosal villi. However, there are significant differences between the results from Kalima et $\mathrm{al}^{33,34}$ and the results presented in the current study. No mortality was observed compared with a reported mortality between $25 \%$ (rats) and $9.5 \%$ (pigs) due to intestinal perforation, paralytic ileus, or purulent peritonitis. In addition, the unique feature of intra-abdominal fistulae could not be recapitulated. When reporting on SLO in pigs, Kalima et $\mathrm{al}^{35}$ described a progression of the inflammatory state as well as a progression of the intra-abdominal lesions during the follow-up period of up to 98 days. In contrast to that, when SLO was performed in rats, a great proportion of the animals showed a reorganization of lymph flow and recovered from the initial inflammatory response. In our model, the time course of the inflammatory state peaked at day 7 after SLO and was followed by a phase of resolution and return to baseline homeostasis between days 21 and 61 . However, this acute model is limited in fully recapitulating the complex pathophysiology of IBD, which develops over several years and is often characterized by phases of remission, followed by acute inflammatory flares.

A sufficient and lasting lymphatic obstruction is key in validating the SLO model we present herein because the development of significant lymphatic collaterals or adaptive lymphatic reorganization in regions of SLO could diminish the anticipated lymphostasis. Inflammation itself elicits lymphangiogenesis by inducing the regrowth of intranodal and extranodal lymph vessels from existing lymphatic networks. ${ }^{42}$ In addition, a growing body of evidence indicates that circulating endothelial progenitor cells might contribute to lymphatic expansion and inflammation-induced remodeling (lymphovasculogenesis). ${ }^{43-45}$ Moreover, an increase in intraluminal pressure after lymphatic obstruction can induce the opening of lymphovenous shunts and bypass collaterals. ${ }^{46,47}$ The results from the lymphography show that all SLO animals (irrespective of the time point) showed a sufficient lymphatic obstruction without any signs of major collaterals, recanalization, or venous bypass. In addition, all SLO animals showed signs of lymphatic stasis and retrograde lymph flow, which is another direct sign of a successful and complete long-lasting lymphatic obstruction. Still, it is reasonable to assume that our model of SLO in AGMs produced an initial severe inflammatory response that was followed by some form of minor lymphatic reorganization.

Although the inflammatory response was characterized by an increase in inflammatory mediators, the recovery phase was characterized by an increase in proresolving mediators. Regarding the different inflammatory profiles after SLO within the ileocecal region, there is evidence for a greater susceptibility of the small bowel to an insufficient lymphatic function. A form of intestinal inflammation in dogs, elicited by spontaneous lipogranulomatous lymphangitis with intestinal lymphangiectasia and proteinlosing enteropathy, is more severe in the small bowel. ${ }^{48,49}$ In addition, gut inflammation with enteric lymphangitis after Chlamydia suis infection in pigs is also restricted to the small bowel. ${ }^{50}$

The pivotal role of the intestinal lymphatic vasculature for the structural integrity and function of the local immune surveillance in the gut was recently shown by two studies using murine conditional ablation models. Jang et $\mathrm{al}^{51}$ used a mouse model of diphtheria toxin-induced ablation of lymphatic vessel endothelial hyaluronan receptor-1, expressing lymphatic vessels in adult mice. Strikingly, 
within 60 hours of diphtheria toxin injection, all ablated animals died of sepsis, presumably because of a loss of structural integrity of intestinal villi with a consecutive breakdown in the local immune surveillance. Davis et al ${ }^{52}$ used a mouse model with deletion of the lymphangiogenic receptor for the adrenomedullin peptide, calcitonin receptor-like receptor, and were able to show that loss of lymphatic calcitonin receptor-like receptor was sufficient to induce intestinal lymphangiectasia, characterized by dilated lacteals and protein-losing enteropathy. Although these mice showed no basal intestinal inflammation, they showed an exacerbated response to an intestinal inflammatory challenge (indomethacin) and, most important, a failure to resolve the inflammatory state. ${ }^{52}$ These results support this concept as regional lymphatic obstruction leads to a regional and systemic inflammatory state. In conclusion, there is a growing body of evidence indicating a pivotal role of the intestinal lymphatic vasculature in the structural and functional integrity of the gut architecture by participating in barrier function, immune cell trafficking, antigen drainage, and, most important, resolution of inflammation.

Resolution of inflammation is a tightly orchestrated process, involving specific endogenous mediators and protective proresolution pathways. These proresolving mediators and pathways can be disrupted in inflammatory conditions, leading to prolonged and exaggerated inflammatory responses. ${ }^{16}$ In this study, the AGMs responded with a severe inflammatory state to SLO (with maximal inflammation at day 7), characterized by increased levels of inflammatory cytokines and chemokines, including TNF- $\alpha$, IL-6, MCP-1, and IL-1 $\beta$; all of these have been shown to be involved in murine and human IBD as well in other AGM models of inflammation. ${ }^{53,54}$ The development of an inflammatory response to SLO was linked with increased levels of the proinflammatory lipid mediators $\mathrm{LTB}_{4}$ and $\mathrm{PGE}_{2}$ in the small bowel and correlated with a decrease in proresolving mediators from all three major bioactive metabolomes (ie, AA, EPA, and DHA) in the colon, identified and quantified using LC-MS/MS-based LM metabololipidomics. $\mathrm{LTB}_{4}$ and $\mathrm{PGE}_{2}$ are biosynthesized during the initial phases of inflammation through enzymatic processing of AA (by cyclooxygenases and lipoxygenases), and they are responsible for neutrophil recruitment to the site of injury and initiation of inflammatory responses. It was considered whether the presence of neutrophils in the intestinal tissues of AGMs at day 7 could represent a major source of the elevated levels of $\mathrm{LTB}_{4}$, as $\mathrm{LTB}_{4}$ is one of the major bioactive products released by activated neutrophils. After transmigration, neutrophils become exposed to increased levels of autacoids (eg, $\mathrm{PGE}_{2}$ ) that alter neutrophil gene expression and phenotype, which, in turn, drives neutrophil expression of lipoxin synthetic enzymes. This LM-SPM class switch increases production of LXs (eg, $\mathrm{LXA}_{4}$ and $\mathrm{LXB}_{2}$ ), to recruit both anti-inflammatory and proresolving programs, in vitro and in vivo ${ }^{55,56}$ In line with this, up-regulated levels of AA-derived lipoxin pathway factors, specifically $\mathrm{LXA}_{4}$ and 5S,15S-diHETE, were found at day 21 , at which time the inflammatory phenotype was declining. It is possible that those signaling pathways that induce $\mathrm{LTB}_{4}$ and $\mathrm{PGE}_{2}$ may actively convert the production of lipid mediators from proinflammatory to proresolving by triggering the production of LXs, protectins, and resolvins, which might hasten resolution of intestinal inflammation in AGMs subjected to SLO; these results are consistent with other inflammatory models. ${ }^{56,57}$

SPMs counterregulate proinflammatory mediator production, including cytokines, such as TNF- $\alpha$ and IL- 6 , and eicosanoids [eg, $\mathrm{LTB}_{4}$ and $\mathrm{TxB} 2$ mean thromboxane $\mathrm{B} 2$ $\left.\left(\mathrm{TxB}_{2}\right)\right]$, thereby promoting resolution of inflammation. ${ }^{58}$ These highly specific regulators are formed via the stereospecific conversion of essential PUFAs by enzymes, such as lipoxygenases. ${ }^{16}$ Herein, we found that at 7 days, AGMs with SLO displayed a heightened inflammatory milieu both systemically and locally within the ileocecal region. In turn, this led to an increase in LM profile, which was followed by a decrease from day 21 to 61 after SLO, demonstrating a process of natural resolution. In addition, it is plausible that endogenous checkpoints are fully functional and operative in AGMs, avoiding further progression of inflammation due to SLO. Our findings herein showing the sequence of active inflammation with decreased levels of SPMs, followed by resolution of inflammation and a respective active SPM profile, show some parallels to the inflammatory state as well as SPM profile of relapsing and remitting disease in human UC. ${ }^{59}$ Furthermore, it was demonstrated that patients with active UC are characterized by diminished SPM metabolism, whereas UC patients undergoing resolution show SPM profiles that more closely resemble the lipid profiles of healthy controls. ${ }^{38}$ Our results demonstrate that animals undergoing SLO show the capability to respond to an inflammatory stimulus with the up-regulation of SPMs, a distinct feature that is possibly defective in UC patients during active disease and, thus, hinders resolution of inflammation and induction of remission.

Profiles and protective roles of SPMs derived from PUFAs, such as DHA and EPA, have already been linked to protective actions in intestinal inflammation, predominantly in murine models of experimental colitis. In line with these reports, a distinct pattern of SPMs derived from all major bioactive metabolomes was observed.

Resolvins and protectins are highly potent lipid mediators that display potent anti-inflammatory and proresolving effects by inhibiting the production of proinflammatory mediators, regulating neutrophil trafficking, stimulating lymphatic drainage of leukocytes, and promoting efferocytosis. ${ }^{16}$ Resolvins are divided into the D series (RvDs; derived from DHAs), six AT analogs of the D series (AT-RvD1-6), and the E series (RvEs; derived from EPAs). Arita et $\mathrm{al}^{30}$ first demonstrated beneficial actions of the E-series resolvins (RvE1), and Ishida et $\mathrm{al}^{29}$ and Campbell et $\mathrm{al}^{20}$ confirmed these results. Focusing on D-series resolvins, Bento et $\mathrm{al}^{31}$ showed that AT-RvD1, its 
precursor 17(R)-hydroxy DHA, and RvD2 were protective in trinitrobenzene sulfonic acid as well as in dextran sodium sulfate colitis. Furthermore, it has been suggested that $\mathrm{PD} 1_{\mathrm{n}-3}$ DPA or $\mathrm{RvD}_{\mathrm{n}-3}$ DPA activate distinct protective responses potentially through the engagement of different receptors, as demonstrated by their different actions in regulating inflammatory cytokines $\left(\mathrm{PD} 1_{\mathrm{n}-3}\right.$ DPA regulated TNF- $\alpha$, IL-1 $\beta$, and IL-6, whereas RvD5 $5_{n-3}$ DPA only partially reduced IL-1 $\beta$ levels). These studies reported an improved disease activity score, reduced colonic damage, and lower neutrophil infiltration after exogenous administration of both D- and E-series resolvins. The results from this study showing an up-regulation of theses endogenous SPMs in AGMs also concur with these earlier findings in rodents. Interestingly, ileocecal changes were observed in $\mathrm{Rv}$ production levels, such as a twofold up-regulation of AT-RvD1， RvD5， RvD6， 10S,17S-diHDPA， 5S,15SdiHETE, and RvE2 at 21 compared with 7 days after SLO compared, underlining an important role of these SPMs in the resolution of intestinal inflammation and suggesting an endogenous protective mechanism for reducing inflammation in AGMs after SLO. Similarly, protectins and maresins, which are generated from DHA from single cells, also showed the same trend. We found an increase in MaR1 and $\mathrm{LXA}_{4}$ during the switch from inflammation to resolution between 7- and 21-day animals after SLO. Both of these SPMs have been previously shown to be protective when administered during intestinal inflammation, ${ }^{32,60}$ where pharmacologic treatment with $\mathrm{LXA}_{4}$ effectively promoted resolution of inflammation in experimental colitis. ${ }^{61}$ In addition, exogenous administration of low doses of $\mathrm{LXA}_{4}$ has been shown to inhibit neutrophil accumulation in postischemic mesenteric tissues ${ }^{62}$ and even remote organs, such as the brain. ${ }^{63,64}$ It is, therefore, likely that increased $\mathrm{LXA}_{4}$ levels were generated by cooperative metabolism between neutrophils, platelets, and resident tissue cells, such as epithelial cells, through human platelet 12-lipoxygenase and neutrophil 5-lipoxygenase. ${ }^{65}$ Consistent with this, a parallel increase was also seen in $\mathrm{LXB}_{4}$, which is a positional isomer of $\mathrm{LXA}_{4}$, a potent mediator and the principal lipoxin species found in mammals. ${ }^{66}$

The concept of using resolvins, lipoxins, protectins, or maresins as a target in models of inflammation (including intestinal inflammation) has been used before, underlining the therapeutic potential of these molecules. ${ }^{67}$ SPMs have been proved to be effective in murine models of arthritis, ${ }^{68}$ human skin inflammation, ${ }^{69}$ and experimental ${ }^{70}$ and human periodontitis ${ }^{71}$; and they have also been shown to lower antibiotic requirements during bacterial infection. ${ }^{72}$ This study provides not only the first identification of SPMs in AGMs, but it is also the first documentation of serum LMSPM during intestinal inflammation in an NHP model. This study reveals a distinct trend toward an up-regulation in SPMs from all major bioactive metabolomes at 21 days after SLO compared with 7-day animals. These results concur with a recent report from a baboon model of pneumococcal pneumonia in which infected animals showed reduced SPM profiles. ${ }^{58}$ After treatment, these animals then displayed restored SPM levels, including resolvins and lipoxins.

Results from both clinical and animal studies indicate that TNF- $\alpha$ plays critical roles in the pathogenesis of IBD. For instance, TNF- $\alpha$-deficient mice and anti-TNF- $\alpha$ treatments reduce intestinal inflammation in several animal models of IBD, ${ }^{73}$ and overexpression of TNF- $\alpha$ in mice provokes the development of a CD-like phenotype. ${ }^{74} \mathrm{TNF}$ $\alpha$ induces NF- $\kappa \mathrm{B}-$ mediated mobilization of endothelial adhesion molecules and inflammatory mediators and can suppress (or enhance) neutrophil apoptosis. ${ }^{75}$ As such, blockade of TNF- $\alpha$ is now a widely applied therapeutic strategy for managing CD. However, recent findings have identified a central role for TNF- $\alpha$ in up-regulating formyl receptor 2 (alias ALX), a central receptor that transduces the actions of proresolving mediators annexin $\mathrm{A} 1, \mathrm{LXA}_{4}$, and $\mathrm{RvD} 1{ }^{76}$ consistent with a dual role for this cytokine. It could be suggested that up-regulation of TNF- $\alpha$ could increase levels of $\mathrm{LXA}_{4}$ and other SPMs in both the ileum (at day 61) and the colon (up-regulation at 21 days after SLO). Clinically, defective $\mathrm{LXA}_{4}$ biosynthesis is observed in colonic mucosa of UC patients, which may explain the increased inflammation observed in these patients. ${ }^{77}$ The increased mucosal biosynthesis of $\mathrm{LXA}_{4}$ (and annexin A1) has also been observed in individuals in medically induced remission from $\mathrm{UC}{ }^{78}$

Interestingly, an increase was found in platelet counts during the inflammatory state 7 days after SLO. It is well known that both UC and CD are characterized by quantitative and functional alterations in platelet characteristics, such as thrombocytosis, as well as heightened platelet activation and aggregation. Besides their established role in thrombosis and coagulation, mounting evidence suggests a crucial role of platelets in amplifying mucosal immune responses by eliciting proinflammatory mediators and amplifying leukocyte recruitment and activation. Furthermore, platelets have now also been linked to the development and function of the lymphatic system as they mediate embryonic blood-lymphatic vascular divergence ${ }^{79}$ and are involved in maintaining lymphovenous hemostasis. ${ }^{80}$ In addition, platelets (via C-type lectin-like receptor 2 signaling) inhibit migration, proliferation, and tube formation of lymphatic endothelial cells by interacting with the surface protein podoplanin; thus, they can inhibit lymphangiogenesis. ${ }^{81}$ This observation becomes even more important under inflammatory conditions in the gut because blocking lymphangiogenesis aggravates murine colitis and induction of lymphangiogenesis ameliorates experimental colitis. Sato et $\mathrm{al}^{13}$ confirmed the presence of platelets in lymphatic vessels of the inflamed intestinal mucosa during murine colitis as well as an increase in platelet count in the lymph collected from the thoracic duct in rats subjected to trinitrobenzene sulfonic acid-induced ileitis. These results suggest that, under inflammatory conditions, platelets can migrate into the intestinal lymphatic vasculature and suppress 
lymphangiogenesis. It is, therefore, possible that the observed thrombocytosis 7 days after SLO has, at least in part, blocked intestinal lymphatic remodeling and, therefore, aggravated the inflammatory state by mitigating immune cell efflux and fluid clearance.

The use of NHPs in experimental basic research is indispensable because they resemble human physiology and immunity much more closely than any other experimental model. Among NHPs, the AGM is a well-accepted experimental model to study innate and acquired immunity. However, most studies report results in the field of viral infection $^{82}$; recently, Hao et al ${ }^{83}$ developed the first NHP model of intestinal inflammation in rhesus macaques by orally administering dextran sodium sulfate. In addition, they used two AGMs to induce chronic colitis. Although their study clearly demonstrates the feasibility of using NHPs in IBD research, every dextran sodium sulfate-based model of chemically induced colitis more closely resembles features of UC than CD as this was shown in the original murine model. Thus, to our knowledge, the present study is the first of its kind to provide data on intestinal inflammation in AGMs with a more CD phenotype. In addition, our results on SPMs are the first report in this species. The growing body of evidence suggesting a crucial involvement of lymphatics in the pathogenesis of CD has generated new interest in animal models to study lymphatic obstruction in relation to intestinal inflammation. Recently, a new animal model involving virus-induced intestinal inflammation in immunodeficient mice was presented, in which a chronicactive lymphangitis results in ileal enteritis as well as colitis. ${ }^{84}$ This model is of particular interest because the lymphatic phenotype (ranging from dilated lymphatics to lymphangitis) precedes the inflammatory phenotype, thus offering the unique possibility to study different stages in the continuum from lymphangitis to intestinal inflammation. The animal model of SLO, which is presented herein, offers some additional opportunities to the research community: it allows the study of inflammation as a consequence of lymphatic failure, and it also generates inflammation in the terminal ileum and not solely in the colon.

New therapeutic strategies promoting endogenous resolution would represent a highly beneficial therapeutic approach in IBD, especially given current treatments (eg, biological agents, such as anti-TNF- $\alpha$, are immunosuppressive, expensive, and often lose effectiveness in many patients). ${ }^{24}$ So far, the available human data consist of trials that used nutritional strategies to supplement SPM precursors, such as essential omega-3 PUFAs. Although some studies suggest beneficial effects, recent meta-analyses found no evidence for the ability of omega-3 PUFAs to induce or maintain remission in $\mathrm{CD}^{85}$ and UC. ${ }^{86}$ However, these findings could be due to problems with the systemic oral delivery (especially in IBD patients with the risk of insufficient intestinal absorption) as well as the potential of these precursors to be metabolized to various SPMs, which might not be effective in controlling intestinal resolution. ${ }^{28}$ However, if SPMs could be delivered locally and timely, they present an attractive future therapeutic avenue to induce remission in IBD patients by aiding the resolution of inflammation without potential immunosuppressive adverse effects. Furthermore, changes in proresolving mediator levels may represent a predictive index of therapeutic efficacy. This novel NHP model provides evidence of the important roles played by patent lymphatic flow in normal gut homeostasis and how lymphatic obstruction is an important, but too often disregarded, component in CD. Furthermore, it provides novel evidence identifying SPMs, including AA-, DPA-, and EPA-derived SPMs, that may regulate intestinal mucosal injury, inflammation, and repair, supporting the resolution of inflammation in the gut of AGMs after SLO.

\section{Supplemental Data}

Supplemental material for this article can be found at http://doi.org/10.1016/j.ajpath.2019.05.013.

\section{References}

1. Ananthakrishnan AN: Epidemiology and risk factors for IBD. Nat Rev Gastroenterol Hepatol 2015, 12:205-217

2. Van Kruiningen HJ, Colombel JF: The forgotten role of lymphangitis in Crohn's disease. Gut 2008, 57:1-4

3. Bernier-Latmani J, Petrova TV: Intestinal lymphatic vasculature: structure, mechanisms and functions. Nat Rev Gastroenterol Hepatol 2017, 14:510-526

4. Alitalo K: The lymphatic vasculature in disease. Nat Med 2011, 17: $1371-1380$

5. Becker F, Yi P, Al-Kofahi M, Ganta VC, Morris J, Alexander JS: Lymphatic dysregulation in intestinal inflammation: new insights into inflammatory bowel disease pathomechanisms. Lymphology 2014, 47:3-27

6. Geleff S, Schoppmann SF, Oberhuber G: Increase in podoplaninexpressing intestinal lymphatic vessels in inflammatory bowel disease. Virchows Arch 2003, 442:231-237

7. Rahier JF, De Beauce S, Dubuquoy L, Erdual E, Colombel JF, JouretMourin A, Geboes K, Desreumaux P: Increased lymphatic vessel density and lymphangiogenesis in inflammatory bowel disease. Aliment Pharmacol Ther 2011, 34:533-543

8. Pedica F, Ligorio C, Tonelli P, Bartolini S, Baccarini P: Lymphangiogenesis in Crohn's disease: an immunohistochemical study using monoclonal antibody D2-40. Virchows Arch 2008, 452:57-63

9. Tonelli P, Martellucci J, Lucchese M, Comin CE, Bergamini C, Pedica F, Bargellini T, Valeri A: Preliminary results of the influence of the in vivo use of a lymphatic dye (patent blue v) in the surgical treatment of Crohn's disease. Surg Innov 2014, 21:381-388

10. Randolph GJ, Bala S, Rahier JF, Johnson MW, Wang PL, Nalbantoglu I, Dubuquoy L, Chau A, Pariente B, Kartheuser A, Zinselmeyer BH, Colombel JF: Lymphoid aggregates remodel lymphatic collecting vessels that serve mesenteric lymph nodes in Crohn disease. Am J Pathol 2016, 186:3066-3073

11. Jurisic G, Sundberg JP, Detmar M: Blockade of VEGF receptor-3 aggravates inflammatory bowel disease and lymphatic vessel enlargement. Inflamm Bowel Dis 2013, 19:1983-1989

12. D'Alessio S, Correale C, Tacconi C, Gandelli A, Pietrogrande G, Vetrano S, Genua M, Arena V, Spinelli A, Peyrin-Biroulet L, 
Fiocchi C, Danese S: VEGF-C-dependent stimulation of lymphatic function ameliorates experimental inflammatory bowel disease. J Clin Invest 2014, 124:3863-3878

13. Sato H, Higashiyama M, Hozumi H, Sato S, Furuhashi H, Takajo T, Maruta K, Yasutake Y, Narimatsu K, Yoshikawa K, Kurihara C, Okada Y, Watanabe C, Komoto S, Tomita K, Nagao S, Miura S, Hokari R: Platelet interaction with lymphatics aggravates intestinal inflammation by suppressing lymphangiogenesis. Am J Physiol Gastrointest Liver Physiol 2016, 311:G276-G285

14. Becker F, Kurmaeva E, Gavins FN, Stevenson EV, Navratil AR, Jin L, Tsunoda I, Orr AW, Alexander JS, Ostanin DV: A critical role for monocytes/macrophages during intestinal inflammation-associated lymphangiogenesis. Inflamm Bowel Dis 2016, 22:1326-1345

15. Becker F, Potepalov S, Shehzahdi R, Bernas M, Witte M, Abreo F, Traylor J, Orr WA, Tsunoda I, Alexander JS: Downregulation of FoxC2 increased susceptibility to experimental colitis: influence of lymphatic drainage function? Inflamm Bowel Dis 2015, 21: $1282-1296$

16. Serhan CN: Pro-resolving lipid mediators are leads for resolution physiology. Nature 2014, 510:92-101

17. Colgan SP, Serhan CN, Parkos CA, Delp-Archer C, Madara JL: Lipoxin A4 modulates transmigration of human neutrophils across intestinal epithelial monolayers. J Clin Invest 1993, 92:75-82

18. Maddox JF, Colgan SP, Clish CB, Petasis NA, Fokin VV, Serhan CN: Lipoxin B4 regulates human monocyte/neutrophil adherence and motility: design of stable lipoxin B4 analogs with increased biologic activity. FASEB J 1998, 12:487-494

19. Serhan CN, Hong S, Gronert K, Colgan SP, Devchand PR, Mirick G, Moussignac RL: Resolvins: a family of bioactive products of omega3 fatty acid transformation circuits initiated by aspirin treatment that counter proinflammation signals. J Exp Med 2002, 196:1025-1037

20. Campbell EL, Louis NA, Tomassetti SE, Canny GO, Arita M, Serhan CN, Colgan SP: Resolvin E1 promotes mucosal surface clearance of neutrophils: a new paradigm for inflammatory resolution. FASEB J 2007, 21:3162-3170

21. Sun YP, Oh SF, Uddin J, Yang R, Gotlinger K, Campbell E, Colgan SP, Petasis NA, Serhan CN: Resolvin D1 and its aspirintriggered 17R epimer: stereochemical assignments, antiinflammatory properties, and enzymatic inactivation. J Biol Chem 2007, 282:9323-9334

22. Bannenberg GL, Chiang N, Ariel A, Arita M, Tjonahen E, Gotlinger KH, Hong S, Serhan CN: Molecular circuits of resolution: formation and actions of resolvins and protectins. J Immunol 2005, 174:4345-4355

23. Serhan CN, Yang R, Martinod K, Kasuga K, Pillai PS, Porter TF, Oh SF, Spite M: Maresins: novel macrophage mediators with potent antiinflammatory and proresolving actions. J Exp Med 2009, 206: $15-23$

24. Gobbetti T, Dalli J, Colas RA, Federici Canova D, Aursnes M, Bonnet D, Alric L, Vergnolle N, Deraison C, Hansen TV, Serhan CN, Perretti M: Protectin D1n-3 DPA and resolvin D5n-3 DPA are effectors of intestinal protection. Proc Natl Acad Sci U S A 2017, 114:3963-3968

25. Fullerton JN, Gilroy DW: Resolution of inflammation: a new therapeutic frontier. Nat Rev Drug Discov 2016, 15:551-567

26. Masoodi M, Pearl DS, Eiden M, Shute JK, Brown JF, Calder PC, Trebble TM: Altered colonic mucosal polyunsaturated fatty acid (PUFA) derived lipid mediators in ulcerative colitis: new insight into relationship with disease activity and pathophysiology. PLoS One 2013, 8:e76532

27. Pearl DS, Masoodi M, Eiden M, Brummer J, Gullick D, McKeever TM, Whittaker MA, Nitch-Smith H, Brown JF, Shute JK, Mills G, Calder PC, Trebble TM: Altered colonic mucosal availability of n-3 and n-6 polyunsaturated fatty acids in ulcerative colitis and the relationship to disease activity. J Crohn's Colitis 2014, 8: $70-79$

28. Ungaro F, Tacconi C, Massimino L, Corsetto PA, Correale C, Fonteyne P, Piontini A, Garzarelli V, Calcaterra F, Della Bella S,
Spinelli A, Carvello M, Rizzo AM, Vetrano S, Petti L, Fiorino G, Furfaro F, Mavilio D, Maddipati KR, Malesci A, Peyrin-Biroulet L, D'Alessio S, Danese S: MFSD2A promotes endothelial generation of inflammation-resolving lipid mediators and reduces colitis in mice. Gastroenterology 2017, 153:1363-1377.e6

29. Ishida T, Yoshida M, Arita M, Nishitani Y, Nishiumi S, Masuda A, Mizuno S, Takagawa T, Morita Y, Kutsumi H, Inokuchi H, Serhan CN, Blumberg RS, Azuma T: Resolvin E1, an endogenous lipid mediator derived from eicosapentaenoic acid, prevents dextran sulfate sodium-induced colitis. Inflamm Bowel Dis 2010, 16:87-95

30. Arita M, Yoshida M, Hong S, Tjonahen E, Glickman JN, Petasis NA, Blumberg RS, Serhan CN: Resolvin E1, an endogenous lipid mediator derived from omega-3 eicosapentaenoic acid, protects against 2,4,6-trinitrobenzene sulfonic acid-induced colitis. Proc Natl Acad Sci U S A 2005, 102:7671-7676

31. Bento AF, Claudino RF, Dutra RC, Marcon R, Calixto JB: Omega-3 fatty acid-derived mediators 17(R)-hydroxy docosahexaenoic acid, aspirin-triggered resolvin D1 and resolvin D2 prevent experimental colitis in mice. J Immunol 2011, 187:1957-1969

32. Marcon R, Bento AF, Dutra RC, Bicca MA, Leite DF, Calixto JB: Maresin 1, a proresolving lipid mediator derived from omega-3 polyunsaturated fatty acids, exerts protective actions in murine models of colitis. J Immunol 2013, 191:4288-4298

33. Kalima TV: Experimental lymphatic obstruction in the ileum. Ann Chir Gynaecol Fenn 1970, 59:187-201

34. Kalima TV, Collan Y: Intestinal villus in experimental lymphatic obstruction: correlation of light and electron microscopic findings with clinical diseases. Scand J Gastroenterol 1970, 5:497-510

35. Kalima TV, Saloniemi H, Rahko T: Experimental regional enteritis in pigs. Scand J Gastroenterol 1976, 11:353-362

36. Colas RA, Shinohara M, Dalli J, Chiang N, Serhan CN: Identification and signature profiles for pro-resolving and inflammatory lipid mediators in human tissue. Am J Physiol Cell Physiol 2014, 307: C39-C54

37. English JT, Norris PC, Hodges RR, Dartt DA, Serhan CN: Identification and profiling of specialized pro-resolving mediators in human tears by lipid mediator metabolomics. Prostaglandins Leukot Essent Fatty Acids 2017, 117:17-27

38. Ungaro F, Rubbino F, Danese S, D'Alessio S: Actors and factors in the resolution of intestinal inflammation: lipid mediators as a new approach to therapy in inflammatory bowel diseases. Front Immunol 2017, 8:1331

39. Heatley RV, Bolton PM, Hughes LE, Owen EW: Mesenteric lymphatic obstruction in Crohn's disease. Digestion 1980, 20: 307-313

40. Wu TF, Carati CJ, Macnaughton WK, von der Weid PY: Contractile activity of lymphatic vessels is altered in the TNBS model of guinea pig ileitis. Am J Physiol Gastrointest Liver Physiol 2006, 291: G566-G574

41. Al-Kofahi M, Becker F, Gavins FN, Woolard MD, Tsunoda I, Wang Y, Ostanin D, Zawieja DC, Muthuchamy M, von der Weid PY, Alexander JS: IL-1beta reduces tonic contraction of mesenteric lymphatic muscle cells, with the involvement of cycloxygenase- 2 and prostaglandin E2. Br J Pharmacol 2015, 172:4038-4051

42. Kim H, Kataru RP, Koh GY: Regulation and implications of inflammatory lymphangiogenesis. Trends Immunol 2012, 33:350-356

43. Lee JY, Park C, Cho YP, Lee E, Kim H, Kim P, Yun SH, Yoon YS: Podoplanin-expressing cells derived from bone marrow play a crucial role in postnatal lymphatic neovascularization. Circulation 2010, 122 : $1413-1425$

44. Kerjaschki D, Huttary N, Raab I, Regele H, Bojarski-Nagy K, Bartel G, Krober SM, Greinix H, Rosenmaier A, Karlhofer F, Wick N, Mazal PR: Lymphatic endothelial progenitor cells contribute to de novo lymphangiogenesis in human renal transplants. Nat Med 2006, 12:230-234

45. Maruyama K, Ii M, Cursiefen C, Jackson DG, Keino H, Tomita M, Van Rooijen N, Takenaka H, D'Amore PA, Stein-Streilein J, 
Losordo DW, Streilein JW: Inflammation-induced lymphangiogenesis in the cornea arises from CD11b-positive macrophages. J Clin Invest 2005, 115:2363-2372

46. Burn JI: Obstructive lymphopathy. Ann R Coll Surg Engl 1968, 42: 93-113

47. Bruna J: Types of collateral lymphatic circulation. Lymphology 1974, 7:61-68

48. Van Kruiningen HJ, Lees GE, Hayden DW, Meuten DJ, Rogers WA: Lipogranulomatous lymphangitis in canine intestinal lymphangiectasia. Vet Pathol 1984, 21:377-383

49. Simmerson SM, Armstrong PJ, Wunschmann A, Jessen CR, Crews LJ, Washabau RJ: Clinical features, intestinal histopathology, and outcome in protein-losing enteropathy in Yorkshire Terrier dogs. J Vet Intern Med 2014, 28:331-337

50. Van Kruiningen HJ: An infectious pig model of Crohn's disease. Inflamm Bowel Dis 2016, 22:2106-2111

51. Jang JY, Koh YJ, Lee SH, Lee J, Kim KH, Kim D, Koh GY, Yoo OJ: Conditional ablation of LYVE-1+ cells unveils defensive roles of lymphatic vessels in intestine and lymph nodes. Blood 2013, 122: $2151-2161$

52. Davis RB, Kechele DO, Blakeney ES, Pawlak JB, Caron KM: Lymphatic deletion of calcitonin receptor-like receptor exacerbates intestinal inflammation. JCI Insight 2017, 2:e92465

53. Schechter ME, Andrade BB, He T, Richter GH, Tosh KW, Policicchio BB, Singh A, Raehtz KD, Sheikh V, Ma D, BroccaCofano E, Apetrei C, Tracy R, Ribeiro RM, Sher A, Francischetti IMB, Pandrea I, Sereti I: Inflammatory monocytes expressing tissue factor drive SIV and HIV coagulopathy. Sci Transl Med 2017, 9. eaam5441

54. Chung S, Cuffe H, Marshall SM, McDaniel AL, Ha JH, Kavanagh K, Hong C, Tontonoz P, Temel RE, Parks JS: Dietary cholesterol promotes adipocyte hypertrophy and adipose tissue inflammation in visceral, but not in subcutaneous, fat in monkeys. Arterioscler Thromb Vasc Biol 2014, 34:1880-1887

55. Sugimoto MA, Sousa LP, Pinho V, Perretti M, Teixeira MM: Resolution of inflammation: what controls its onset? Front Immunol 2016, 7:160

56. Serhan CN, Chiang N, Van Dyke TE: Resolving inflammation: dual anti-inflammatory and pro-resolution lipid mediators. Nat Rev Immunol 2008, 8:349-361

57. Serhan CN, Yacoubian S, Yang R: Anti-inflammatory and proresolving lipid mediators. Annu Rev Pathol 2008, 3:279-312

58. Dalli J, Kraft BD, Colas RA, Shinohara M, Fredenburgh LE, Hess DR, Chiang N, Welty-Wolf K, Choi AM, Piantadosi CA, Serhan CN: The regulation of proresolving lipid mediator profiles in baboon pneumonia by inhaled carbon monoxide. Am J Respir Cell Mol Biol 2015, 53:314-325

59. Cosnes J, Gower-Rousseau C, Seksik P, Cortot A: Epidemiology and natural history of inflammatory bowel diseases. Gastroenterology 2011, 140:1785-1794

60. Gewirtz AT, Collier-Hyams LS, Young AN, Kucharzik T, Guilford WJ, Parkinson JF, Williams IR, Neish AS, Madara JL: Lipoxin a4 analogs attenuate induction of intestinal epithelial proinflammatory gene expression and reduce the severity of dextran sodium sulfate-induced colitis. J Immunol 2002, 168:5260-5267

61. Fiorucci S, Wallace JL, Mencarelli A, Distrutti E, Rizzo G, Farneti S, Morelli A, Tseng JL, Suramanyam B, Guilford WJ, Parkinson JF: A beta-oxidation-resistant lipoxin A4 analog treats hapten-induced colitis by attenuating inflammation and immune dysfunction. Proc Natl Acad Sci U S A 2004, 101:15736-15741

62. Brancaleone V, Gobbetti T, Cenac N, le Faouder P, Colom B, Flower RJ, Vergnolle N, Nourshargh S, Perretti M: A vasculo-protective circuit centered on lipoxin A4 and aspirin-triggered 15-epi-lipoxin A4 operative in murine microcirculation. Blood 2013, 122:608-617

63. Gavins FN, Yona S, Kamal AM, Flower RJ, Perretti M: Leukocyte antiadhesive actions of annexin 1: ALXR- and FPR-related anti-inflammatory mechanisms. Blood 2003, 101:4140-4147

64. Vital SA, Becker F, Holloway PM, Russell J, Perretti M, Granger DN, Gavins FN: Formyl-peptide receptor 2/3/lipoxin A4 receptor regulates neutrophil-platelet aggregation and attenuates cerebral inflammation: impact for therapy in cardiovascular disease. Circulation 2016, 133:2169-2179

65. Serhan CN, Sheppard KA: Lipoxin formation during human neutrophil-platelet interactions: evidence for the transformation of leukotriene A4 by platelet 12-lipoxygenase in vitro. J Clin Invest 1990, 85:772-780

66. Livne-Bar I, Wei J, Liu HH, Alqawlaq S, Won GJ, Tuccitto A, Gronert K, Flanagan JG, Sivak JM: Astrocyte-derived lipoxins A4 and B4 promote neuroprotection from acute and chronic injury. J Clin Invest 2017, 127:4403-4414

67. Serhan CN, Levy BD: Resolvins in inflammation: emergence of the pro-resolving superfamily of mediators. J Clin Invest 2018, 128: $2657-2669$

68. Arnardottir HH, Dalli J, Norling LV, Colas RA, Perretti M, Serhan CN: Resolvin D3 is dysregulated in arthritis and reduces arthritic inflammation. J Immunol 2016, 197:2362-2368

69. Motwani MP, Colas RA, George MJ, Flint JD, Dalli J, RichardLoendt A, De Maeyer RP, Serhan CN, Gilroy DW: Pro-resolving mediators promote resolution in a human skin model of UV-killed Escherichia coli-driven acute inflammation. JCI Insight 2018, 3. e94463

70. Van Dyke TE, Hasturk H, Kantarci A, Freire MO, Nguyen D, Dalli J, Serhan CN: Proresolving nanomedicines activate bone regeneration in periodontitis. J Dent Res 2015, 94:148-156

71. Wang CW, Colas RA, Dalli J, Arnardottir HH, Nguyen D, Hasturk H, Chiang N, Van Dyke TE, Serhan CN: Maresin 1 biosynthesis and proresolving anti-infective functions with human-localized aggressive periodontitis leukocytes. Infect Immun 2015, 84:658-665

72. Chiang N, Fredman G, Backhed F, Oh SF, Vickery T, Schmidt BA, Serhan CN: Infection regulates pro-resolving mediators that lower antibiotic requirements. Nature 2012, 484:524-528

73. Neurath MF, Fuss I, Pasparakis M, Alexopoulou L, Haralambous S, Meyer zum Buschenfelde KH, Strober W, Kollias G: Predominant pathogenic role of tumor necrosis factor in experimental colitis in mice. Eur J Immunol 1997, 27:1743-1750

74. Kontoyiannis D, Boulougouris G, Manoloukos M, Armaka M, Apostolaki M, Pizarro T, Kotlyarov A, Forster I, Flavell R, Gaestel M, Tsichlis P, Cominelli F, Kollias G: Genetic dissection of the cellular pathways and signaling mechanisms in modeled tumor necrosis factor-induced Crohn's-like inflammatory bowel disease. J Exp Med 2002, 196:1563-1574

75. van den Berg JM, Weyer S, Weening JJ, Roos D, Kuijpers TW: Divergent effects of tumor necrosis factor alpha on apoptosis of human neutrophils. J Leukoc Biol 2001, 69:467-473

76. Gobbetti T, Coldewey SM, Chen J, McArthur S, le Faouder P, Cenac N, Flower RJ, Thiemermann C, Perretti M: Nonredundant protective properties of FPR2/ALX in polymicrobial murine sepsis. Proc Natl Acad Sci U S A 2014, 111:18685-18690

77. Mangino MJ, Brounts L, Harms B, Heise C: Lipoxin biosynthesis in inflammatory bowel disease. Prostaglandins Other Lipid Mediat 2006, 79:84-92

78. Vong L, Ferraz JG, Dufton N, Panaccione R, Beck PL, Sherman PM, Perretti M, Wallace JL: Up-regulation of annexin-A1 and lipoxin $\mathrm{A}(4)$ in individuals with ulcerative colitis may promote mucosal homeostasis. PLoS One 2012, 7:e39244

79. Bertozzi CC, Schmaier AA, Mericko P, Hess PR, Zou Z, Chen M, Chen CY, Xu B, Lu MM, Zhou D, Sebzda E, Santore MT, Merianos DJ, Stadtfeld M, Flake AW, Graf T, Skoda R, Maltzman JS, Koretzky GA, Kahn ML: Platelets regulate lymphatic vascular development through CLEC-2-SLP-76 signaling. Blood 2010, 116:661-670

80. Hess PR, Rawnsley DR, Jakus Z, Yang Y, Sweet DT, Fu J, Herzog B, Lu M, Nieswandt B, Oliver G, Makinen T, Xia L, Kahn ML: Platelets mediate lymphovenous hemostasis to maintain blood-lymphatic separation throughout life. J Clin Invest 2014, $124: 273-284$

81. Osada M, Inoue O, Ding G, Shirai $\mathrm{T}$, Ichise H, Hirayama K, Takano K, Yatomi Y, Hirashima M, Fujii H, Suzuki-Inoue K, 
Ozaki Y: Platelet activation receptor CLEC-2 regulates blood/lymphatic vessel separation by inhibiting proliferation, migration, and tube formation of lymphatic endothelial cells. J Biol Chem 2012, 287: $22241-22252$

82. Huot N, Jacquelin B, Garcia-Tellez T, Rascle P, Ploquin MJ, Madec Y, Reeves RK, Derreudre-Bosquet N, Muller-Trutwin M: Natural killer cells migrate into and control simian immunodeficiency virus replication in lymph node follicles in African green monkeys. Nat Med 2017, 23:1277-1286

83. Hao XP, Lucero CM, Turkbey B, Bernardo ML, Morcock DR, Deleage C, Trubey CM, Smedley J, Klatt NR, Giavedoni LD, Kristoff J, Xu A, Del Prete GQ, Keele BF, Rao SS, Alvord WG, Choyke PL, Lifson JD, Brenchley JM, Apetrei C, Pandrea I, Estes JD: Experimental colitis in SIV-uninfected rhesus macaques recapitulates important features of pathogenic SIV infection. Nat Commun 2015, 6:8020

84. Seamons A, Treuting PM, Meeker S, Hsu C, Paik J, Brabb T, Escobar SS, Alexander JS, Ericsson AC, Smith JG, MaggioPrice L: Obstructive lymphangitis precedes colitis in murine norovirus-infected Stat1-deficient mice. Am J Pathol 2018, 188: $1536-1554$

85. Turner D, Zlotkin SH, Shah PS, Griffiths AM: Omega 3 fatty acids (fish oil) for maintenance of remission in Crohn's disease. Cochrane Database Syst Rev 2007, 2:CD006320

86. Turner D, Shah PS, Steinhart AH, Zlotkin S, Griffiths AM: Maintenance of remission in inflammatory bowel disease using omega-3 fatty acids (fish oil): a systematic review and meta-analyses. Inflamm Bowel Dis 2011, 17:336-345 